\title{
ENSAYES ANTE CARGAS LATERALES CÍCLICAS REVERSIBLES DE UN EDIFICIO PREFABRICADO DE CONCRETO REFORZADO DE DOS NIVELES. PARTE II: EVALUACIÓN DEL COMPORTAMIENTO DE CONEXIONES PREFABRICADAS Y DEL SISTEMA DE PISO. RECOMENDACIONES DE DISEÑO
}

\author{
Mario E. Rodríguez ${ }^{(l)}$ y John J. Blandón ${ }^{(1)}$
}

\begin{abstract}
RESUMEN
En este estudio se analiza el comportamiento sísmico de conexiones trabe-columna, columnacimentación, así como del sistema de piso, observado durante ensayes experimentales en una estructura de concreto prefabricada, construida a escala un medio con el sistema estructural llamado dual (combinación de muros estructurales de concreto reforzado y marcos). La estructura se ensayó hasta su falla bajo cargas laterales que representan acciones sísmicas. Todos los elementos estructurales, trabes, columnas, muros, sistema de piso e incluso la cimentación fueron prefabricados. Como resultado de esta investigación se dan recomendaciones para el diseño de las conexiones empleadas, así como para el diseño del sistema de piso prefabricado.
\end{abstract}

\begin{abstract}
This paper describes research on the seismic response of a type of precast beam-column connection, column-foundation connection and diaphragms in precast concrete buildings. These structural elements are part of a half scale, two-story precast structure built with a dual structural system (combination of structural walls and frames). This structure was subjected to simulated seismic loading until the structure reached failure. Seismic design criteria and code implications for connections and diaphragms in precast concrete buildings resulting from this research are discussed in the paper.
\end{abstract}

\section{INTRODUCCIÓN}

Este estudio es parte de una investigación en la que en laboratorio se ensayó una estructura prefabricada de concreto reforzado de dos niveles, a escala un medio, la que se sometió a cargas laterales cíclicas reversibles que trataban de simular acciones sísmicas. La descripción general del estudio experimental, así como la evaluación del comportamiento global del espécimen ha sido llevada a cabo en un artículo anterior (Rodríguez y Blandón, 2003). En el presente artículo se

Artículo recibido el 18 de julio de 2002 y aprobado para su publicación el 27 de marzo de 2003. Se aceptarán comentarios y/o discusiones hasta cinco meses después de su publicación.

(1) Instituto de Ingeniería, UNAM, Ciudad Universitaria, Apartado Postal 70-290 Coyoacán, CP 04510, México, DF mrod@servidor.unam.mx ,jblandonv@iingen.unam.mx 
hace énfasis en la evaluación durante el ensaye del comportamiento observado tanto de elementos estructurales, como de sus conexiones, con base en este comportamiento se dan algunas recomendaciones de diseño.

En la construcción de estructuras prefabricadas en México, en aproximadamente los últimos cinco años se ha empezado a usar un tipo de conexión trabe-columna identificada como tipo "ventana". En estos casos, en las columnas prefabricadas de varios niveles se deja una ventana por nivel, por la que atraviesa posteriormente en sitio el refuerzo negativo, así como ganchos del refuerzo positivo. Debido a que estos ganchos en muchas ocasiones no tienen la longitud de desarrollo requerida por reglamentos, en sitio se colocan estribos alrededor de estos ganchos, con el propósito de dar continuidad a este refuerzo. Existe incertidumbre sobre la eficiencia estructural de esta práctica constructiva, lo que es motivo de esta investigación. También es de interés estudiar si a pesar de las limitaciones mencionadas de esta conexión, es posible su empleo en sistemas estructurales del tipo dual, en el cual la mayor parte de las fuerzas sísmicas es resistida por los muros estructurales del sistema. Como resultado de este estudio se dan recomendaciones para el empleo de las conexiones estudiadas en edificaciones prefabricadas.

Un aspecto del diseño sísmico de edificaciones prefabricadas que ha mostrado evidencias de la necesidad de ser estudiado, especialmente después del sismo de Northridge de 1994, es el referente a los criterios de diseño de sistemas de pisos prefabricados para edificaciones en zonas sísmicas. En esta investigación se analiza el comportamiento observado del sistema de piso prefabricado del espécimen durante el ensaye efectuado en esta investigación, así como se estudia su comportamiento y modo de falla. Con base en los resultados de este estudio se propone un procedimiento de diseño para sistemas de piso prefabricados en zonas sísmicas.

Otro problema que ha sido poco estudiado en zonas sísmicas se refiere al comportamiento sísmico de cimentaciones prefabricadas, en este caso del tipo "candelero". Este tipo de cimentación es bastante empleada en estructuras prefabricadas en México. El proceso constructivo se basa en que en sitio la columna prefabricada se inserta dentro de un espacio previamente construido en la cimentación, para posteriormente llenar la junta con mortero. Existen dudas referentes a si este tipo de cimentación tiene un comportamiento semejante al de cimentaciones coladas en sitio.

\section{DESCRIPCIÓN DE LA CONEXIÓN TRABE-COLUMNA PREFABRICADA QUE SE ESTUDIA}

\section{Conexión trabe-columna que se estudia}

La conexión trabe-columna que se estudia en esta investigación se basa en ideas desarrolladas en otros países. La fig. 1 muestra algunas de las características de este tipo de conexiones, las cuales fueron ensayadas en China ante cargas laterales cíclicas reversibles (Restrepo et al., 1989). Las conexiones con los detalles mostrados en la fig. 1a presentaron fallas por adherencia en estos 
ensayes experimentales, mientras que las conexiones con los detalles mostrados en la Fig. 1b mostraron un mejor comportamiento (Restrepo et al., 1989).

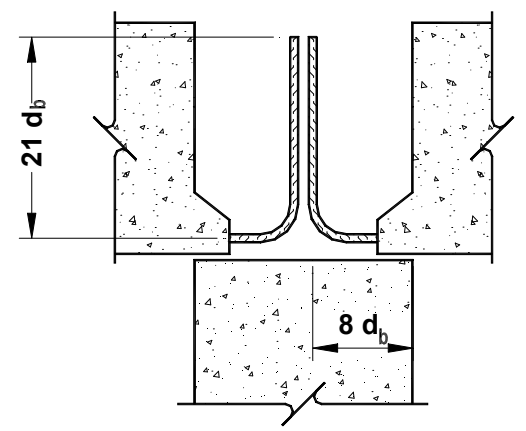

a)

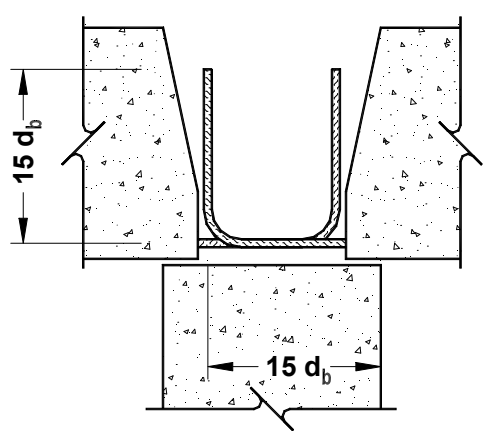

b)

Figura 1. Conexión viga-columna en China (Restrepo et al., 1989)

La conexión tipo "ventana" que se ha empleado en México y que se estudia en esta investigación tiene características semejantes a las mostradas en la fig. 1a. La característica más relevante de este tipo de conexión es que el refuerzo de lecho inferior generalmente no cumple con la longitud de desarrollo requerida por reglamento. Como un intento de superar esta deficiencia, en la modalidad de conexión que aquí se estudia, en algunos casos en la práctica en México se ha propuesto conectar el refuerzo del lecho inferior por medio de estribos interiores; además, se colocan ganchos a través de ductos dejados en los extremos de las vigas con el fin de proporcionar confinamiento en los nudos como se muestra en la fig. 2 (Carranza, 1997). Estos detalles corresponden a una obra construida en México. La conexión mencionada ha sido ensayada en laboratorio ante cargas laterales cíclicas reversibles (Alcocer et al., 2000; Pérez et al., 1998). El comportamiento observado en estos ensayes en la trabe, en la condición de momento positivo a cara de columna, con refuerzo inferior en tensión, mostró que este refuerzo no alcanzó la fluencia. Además, se observó una grieta considerable (alrededor de $15 \mathrm{~mm}$ ) en la interfase trabe prefabricada-columna, lo que sugiere un efecto importante de deslizamiento de esta trabe respecto a la columna. Así mismo, las mediciones de deformímetros eléctricos en los estribos cerrados alrededor de los ganchos del caso estudiado mostraron que éstos no lograron desarrollar un comportamiento inelástico, lo que indica que no pudieron resolver el problema de falta de adherencia del refuerzo.

El comportamiento de la conexión mostrada en la fig. 2 depende del tamaño de columna, ya que si su dimensión es mayor que la longitud de desarrollo del refuerzo de trabe de lecho inferior, podría tener un comportamiento similar al de conexiones coladas en sitio. Sin embargo, en la mayor parte de casos esto llevaría a dimensiones excesivas de columnas. En edificios a base de marcos, el comportamiento de este tipo de conexión es relevante para lograr un comportamiento sísmico adecuado. Sin embargo, en los casos en que se combinen marcos y un número suficiente de muros estructurales (sistema dual), las demandas de deformaciones en las conexiones prefabricadas trabe-columna de este tipo de sistema estructural sometido a sismos intensos, pueden ser bastante menores que las que ocurrirían en eventos sísmicos similares en sistemas estructurales a base de marcos. Estas características sugieren que cuando se cuente con este tipo de conexiones en sistemas duales, puede ser innecesario requisitos especiales de diseño 
sísmico como los requeridos en conexiones que son parte de sistemas estructurales a base de marcos. Este aspecto es estudiado en esta investigación.

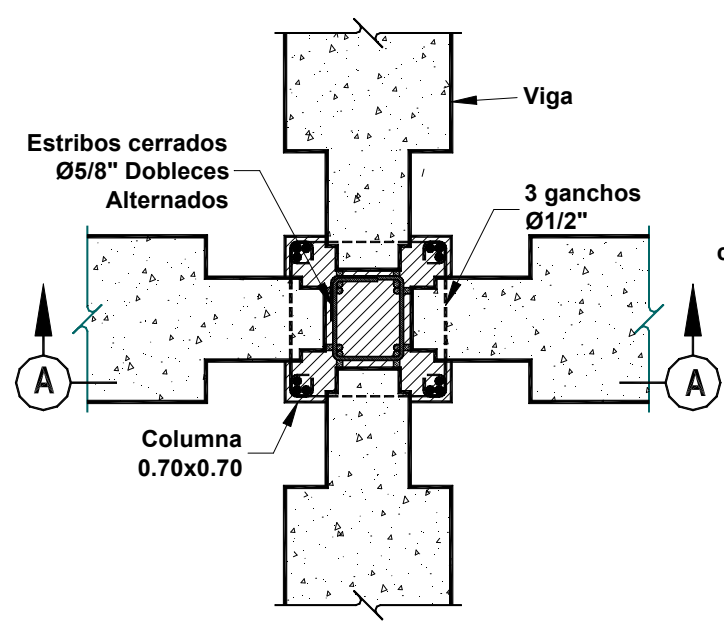

PLANTA

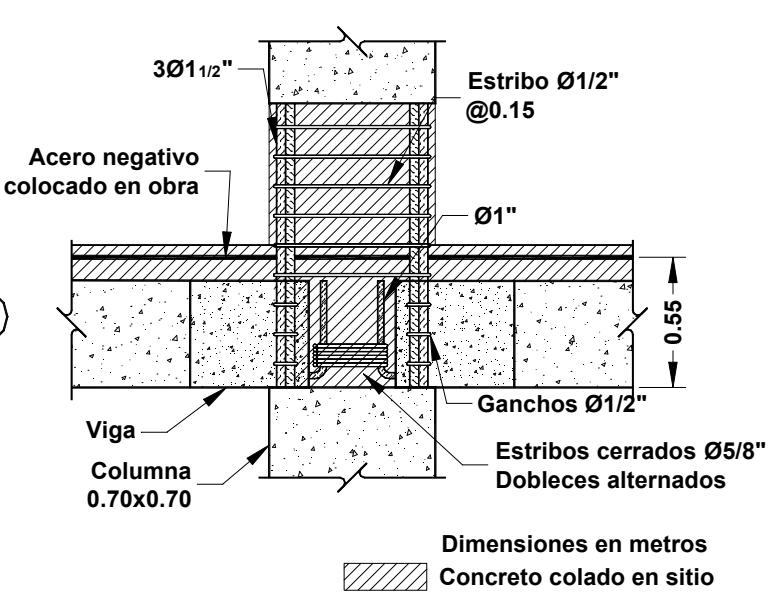

SECCION A-A

Figura 2. Conexión viga-columna (Carranza, 1997)

\section{Descripción de las conexiones trabe-columna estudiadas}

Las conexiones trabe-columna del espécimen estudiadas en los ensayes experimentales llevados a cabo en esta investigación, han sido descritas en el artículo compañero de éste (Rodríguez y Blandón, 2003) y siguen los conceptos ilustrados en las figs 1 y 2. Como se ha mencionado en el artículo anterior, la diferencia de peraltes de las trabes portantes (sentido perpendicular a la dirección de las cargas aplicadas) y de las trabes de borde (orientadas en el sentido de aplicación de cargas, Rodríguez y Blandón, 2003), hizo posible que el refuerzo del lecho inferior llegue a la cara más lejana de las columnas, con lo cual logró la longitud de desarrollo requerida. Una situación diferente se presentó para el refuerzo del lecho inferior de las trabes del eje central del espécimen, en la zona de la conexión trabe-columna. En este refuerzo no fue posible lograr la longitud de desarrollo requerida de reglamentos.

\section{COMPORTAMIENTO DE CONEXIONES TRABE-COLUMNA OBSERVADO EN EL ENSAYE}

El análisis de deformaciones en el refuerzo longitudinal de trabes del espécimen, en las secciones críticas de éstas (conexión trabe-columna), así como las mediciones de curvaturas en estas secciones (Rodríguez y Blandón, 2002), mostró que las demandas de elementos mecánicos no fueron relevantes. Como se ha descrito (Rodríguez y Blandón, 2003), esto fue posible debido a que la mayor parte de las fuerzas laterales aplicadas en el espécimen fueron resistidas por los muros existentes. Posterior al deterioro de estos muros, las conexiones trabe-columna tuvieron 
una mayor contribución en la capacidad resistente de la estructura, con un comportamiento inelástico moderado que permitió la formación de articulaciones incipientes en las trabes.

El marco central, con conexión trabe-columna con longitud insuficiente de desarrollo del refuerzo longitudinal de lecho inferior, mostró un comportamiento que se puede considerar inadecuado desde el punto de vista de comportamiento sísmico, ya que se observó que en esta conexión, para el caso de momento positivo, no tuvo una capacidad relevante de disipación de energía. En este caso, como se comenta posteriormente, el refuerzo del lecho inferior de la viga $\mathrm{T}$ a cara de columna, sólo alcanzó la fluencia cuando la estructura alcanzó gran deterioro, lo cual ocurrió principalmente por el pandeo del refuerzo longitudinal de los muros en sus extremos a nivel cimentación, así como por ruptura de la malla del firme en la vecindad con estos muros. También se observó que el mencionado refuerzo presentó evidencias de falta de adherencia, como se aprecia en la fig. 3. Este comportamiento se analiza en lo que sigue.

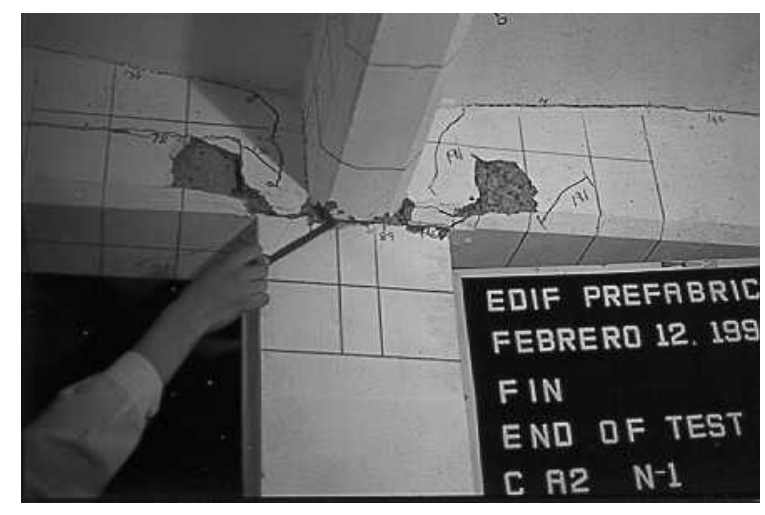

Figura 3. Daño observado al final del ensaye en la conexión trabe-columna de eje central

La fig. 4 muestra resultados de desplazamientos medidos en el refuerzo longitudinal, en el sentido de éste, para las trabes de borde y central, en las secciones instrumentadas a cara de columna, $S_{L}$. También esta figura muestra la forma de medición de estos desplazamientos. La fig. 4a muestra esquemáticamente la ubicación de los instrumentos con los cuales se evaluaron las componentes de la rotación total de la sección instrumentada, $\theta_{T}$. Los instrumentos señalados con $F$ en la fig. 4a registraron desplazamientos con los cuales es posible calcular la rotación de la sección de trabe en la vecindad a cara de columna, $\theta_{F}$. Esta rotación es combinación del deslizamiento de la barra de refuerzo si hay problemas de anclaje, $S_{S}$, de la deformación del refuerzo longitudinal en la trabe dentro de la columna, $S_{P}$, así como de la deformación de este refuerzo en la longitud medida por el instrumento $F, S_{b}$, (fig. 4a). Para el caso de trabes de borde esta longitud fue aproximadamente igual a $0.5 \cdot h$, y para la trabe central esta longitud fue $0.25 \cdot h$, donde h es la altura de la sección. Esto sugiere que los instrumentos señalados con $F$ en la fig. 4a miden rotaciones debido a la combinación de efectos $S_{S}, S_{P}$, y $S_{b}$. Esto dificulta el aislar en estas mediciones la contribución de $S_{S}$. Sin embargo, a pesar de esta limitación, en lo que sigue, en los resultados que se analizan se pretende evaluar esta contribución.

Con las mediciones de los instrumentos señalados como $P$ en la fig. $4 \mathrm{a}$, fue posible calcular la curvatura $\phi_{p}$ de la sección. A partir de esta curvatura y la longitud plástica $l_{p}$, se pudo 
obtener la rotación plástica de la sección, $\theta_{P}$. El análisis de la información obtenida con los instrumentos $P$ se describe en (Rodríguez y Blandón, 2002), sus resultados sirvieron para conocer mejor el comportamiento inelástico durante el ensaye de las secciones críticas instrumentadas.

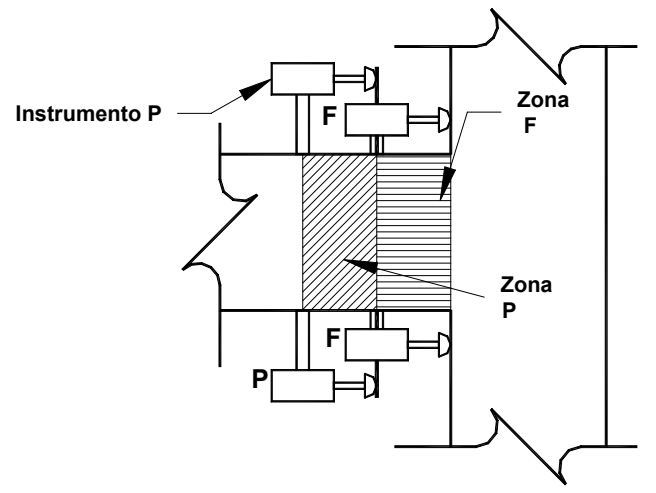

a) Medición de rotaciones

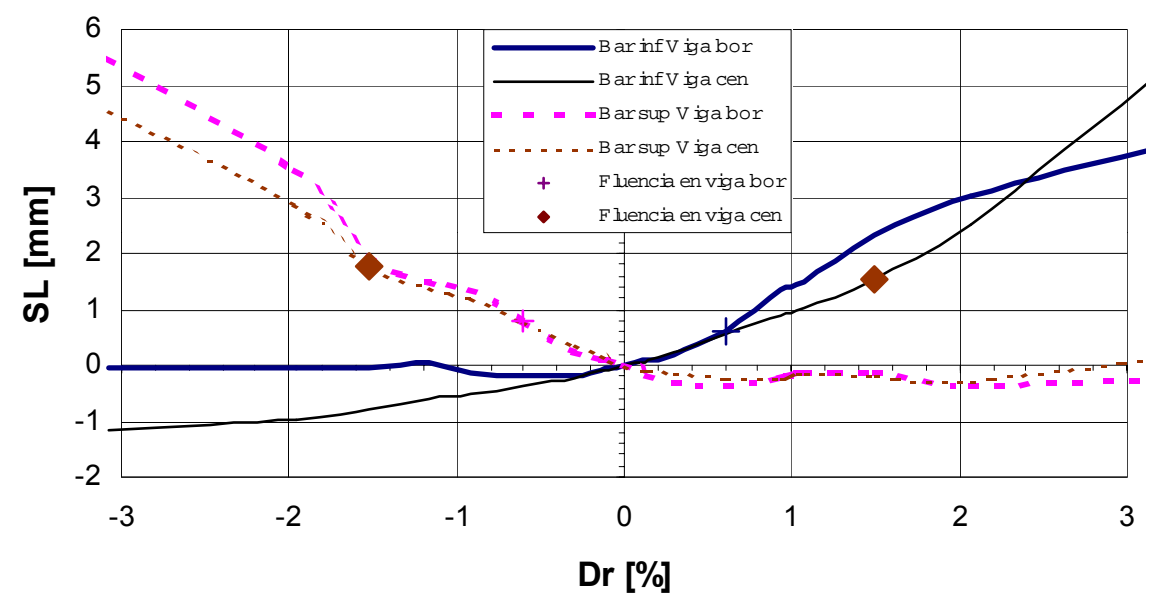

b) Deslizamiento en vigas del nivel 1

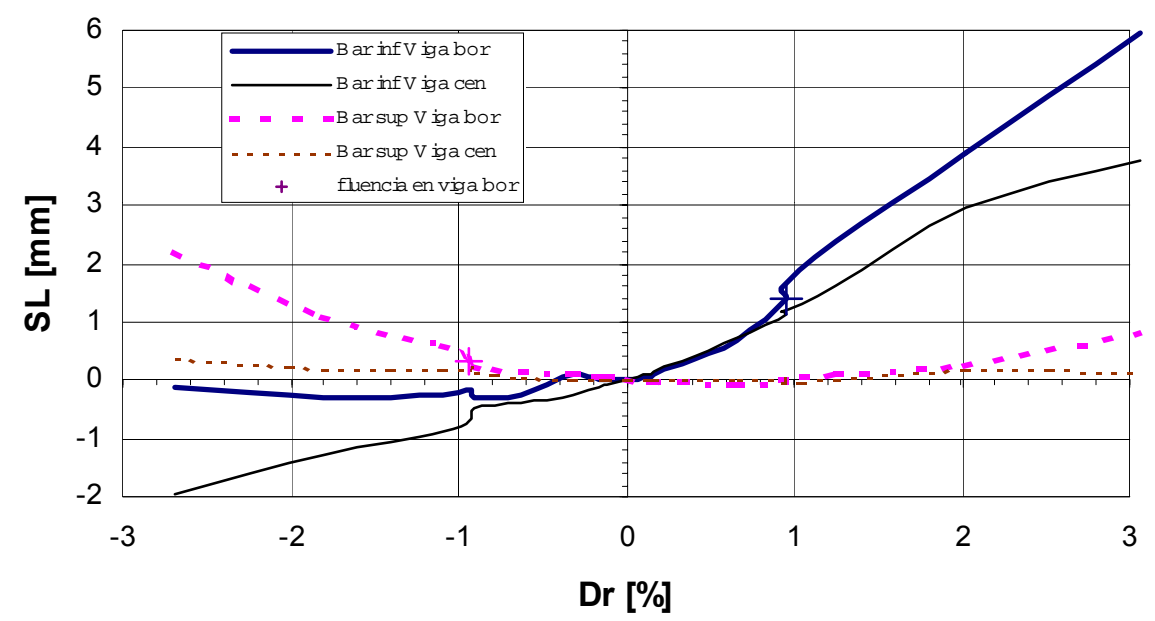

c) Deslizamiento en vigas del nivel 2

Figura 4 Comportamiento de trabes perfabricadas en la zona de conexión trabe-columna 


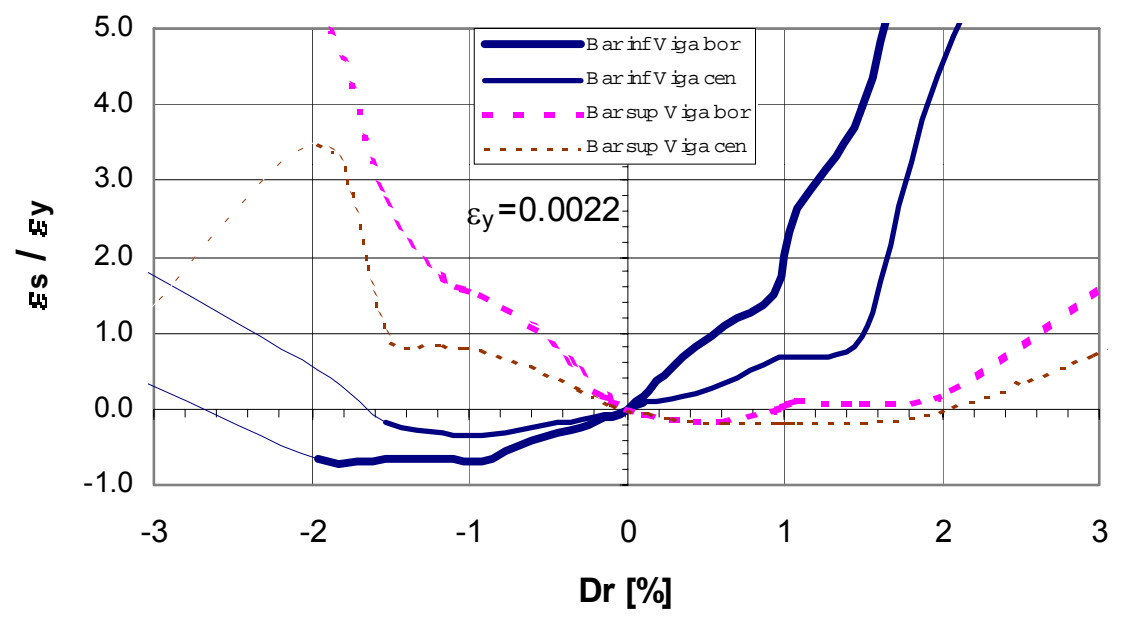

d) Deformaciones medidas en el acero de refuerzo en vigas del nivel 1

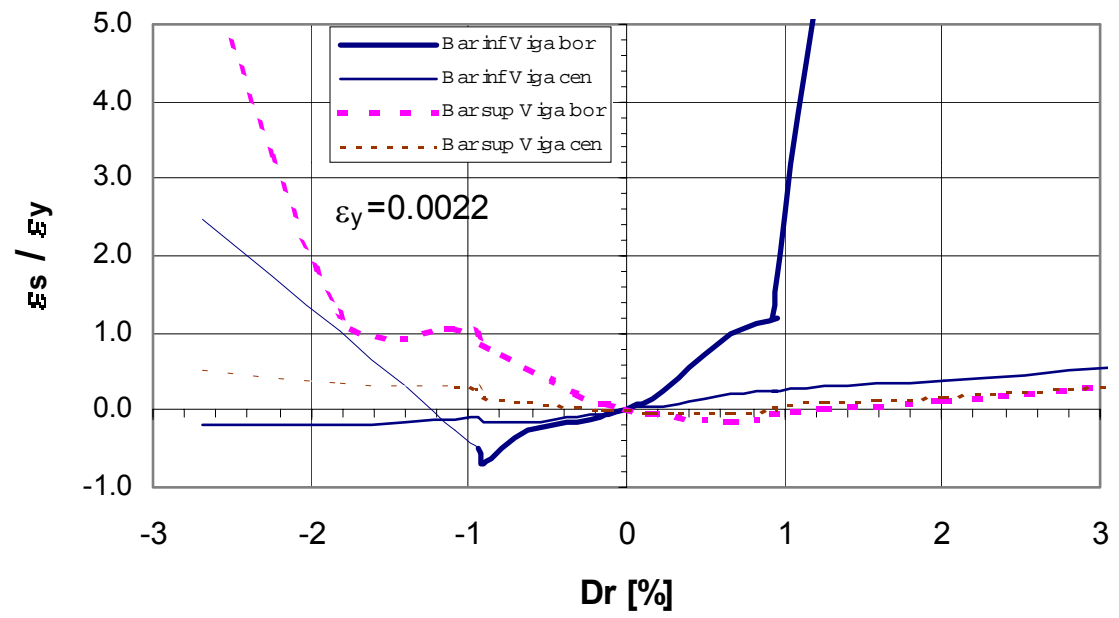

e) Deformaciones medidas en el acero de refuerzo en vigas del nivel 2

Figura 4. Comportamiento de trabes perfabricadas en la zona de conexión trabe-columna (continuación)

Las figs. $4 \mathrm{~b}$ y $4 \mathrm{c}$ muestran los desplazamientos $S_{L}$ medidos para las barras de refuerzo en las secciones de trabes en la vecindad a cara de columna, en los niveles 1 y 2 , respectivamente, tanto para las barras de refuerzo del lecho superior, como las del lecho inferior. Estas últimas, en el eje central del espécimen tienen sólo el 50\% de la longitud de anclaje que especifica las Normas Técnicas Complementarias para Diseño y Construcción de Estructuras de Concreto del DF de 1996 (NTC96). De los resultados mostrados en las mencionadas figuras se observa una característica de interés a la cual se le puede asociar el fenómeno de deslizamiento de la barra, originado por longitud inadecuada de longitud de desarrollo. Como se observa en las figs. $4 \mathrm{~b}$ y 4c, cuando los desplazamientos $S_{L}$ medidos en las barras pasan del caso en tensión ( + ) al de compresión (-), los desplazamientos medidos en compresión son prácticamente nulos, excepto en el caso de las barras de lecho inferior de la conexión con longitud de desarrollo insuficiente (barra lecho inferior de trabe en eje central). Este movimiento (valor negativo de $S_{L}$ ) sugieren daños en el concreto, en la vecindad del gancho, así como deslizamiento de la barra por longitud insuficiente de ésta. 
Los daños mencionados se pueden explicar con la ayuda de la fig. 5, la cual muestra una posible distribución de esfuerzos actuantes en una barra de refuerzo en forma de gancho sometida a tensión, cuando ésta tiene una longitud insuficiente de desarrollo (Minor y Jirsa, 1975). Como se aprecia en la fig. 5, en esta barra curva, la barra tiende a desprenderse del concreto en la zona exterior de la curva, así como en la zona interior de la parte recta del extremo del gancho. Esto origina no sólo una reducción de la capacidad resistente por adherencia, sino también concentraciones de esfuerzo de compresión que pueden llevar al aplastamiento del concreto (Minor y Jirsa, 1975), ambos efectos producen deslizamiento de la barra. Esto explica los mencionados desplazamientos negativos de $S_{L}$ mostrados en las figs $4 \mathrm{~b}$ y $4 \mathrm{c}$, para los casos de las barras con longitud de desarrollo insuficiente. Estos desplazamientos serían entonces debido principalmente a deslizamiento de la barra de refuerzo por problemas de anclaje, $S_{S}$.

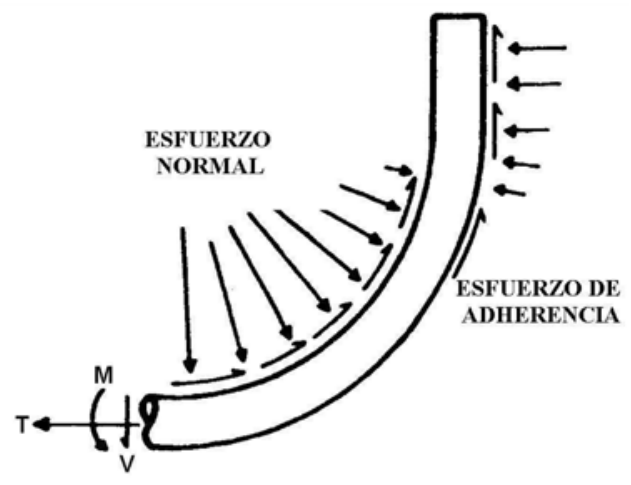

Figura 5. Falla por adherencia en una barra a tensión en forma de gancho

Las figs. 4d y 4e muestran deformaciones medidas en deformímetros adheridos en el refuerzo longitudinal de trabes en secciones de éstas a cara de columnas, tanto en tensión como en compresión, para los niveles 1 y 2 , respectivamente. Estos deformímetros se ubicaron en las trabes a una distancia a cara de columna que varió de $40 \mathrm{~mm}$ a $50 \mathrm{~mm}$. Las deformaciones medidas que se muestran en estas figuras están en función de la deformación relativa global $D r$.

Como se aprecia en las figuras $4 \mathrm{~d}$ y $4 \mathrm{e}$, las deformaciones en las varillas sin suficiente longitud de desarrollo (refuerzo en lecho inferior de vigas en eje central) inician la fluencia en tensión (+) para niveles de deformaciones relativas globales, $\mathrm{Dr}$, bastante altas para el caso nivel 1 (fig. 4d), aproximadamente igual a 0.015 , es decir para niveles de daños severos en el espécimen. En el segundo nivel este refuerzo no llegó a la fluencia (fig. 4e).

Las figuras $4 \mathrm{~d}$ y $4 \mathrm{e}$ muestran que las deformaciones en tensión del refuerzo longitudinal, en el lecho inferior de la trabe del eje central a cara de columna (con longitud de desarrollo inadecuada), son menores que las de este refuerzo en las trabes de borde. Además, estas figuras muestran que en el intervalo de aplicación de cargas asociadas a deformaciones en compresión del refuerzo longitudinal arriba mencionado, las deformaciones en compresión del refuerzo del lecho inferior de la viga central son menores que las registradas para este refuerzo en la viga lateral para ambos niveles del espécimen; mientras que para el refuerzo con longitudes de anclaje requeridas por reglamento (refuerzo en lecho superior en vigas de borde y central), las deformaciones en compresión son comparables. 
Este comportamiento se puede explicar con la ayuda de la fig. 5, con un razonamiento similar al descrito para explicar el problema del deslizamiento en una barra con longitud insuficiente de desarrollo. De acuerdo con la fig. 5, cuando el gancho se somete a tensión, éste tiende a apartarse del concreto, transfiriendo la carga aplicada en la barra principalmente por aplastamiento del concreto en las zonas de la barra indicadas en la fig. 5. Para inversión de la carga, cuando el gancho trabaja en compresión, las partes del gancho indicadas en la fig. 5 con reacciones en compresión en el concreto, debieran tender a separarse para definir diferentes zonas del gancho en compresión, con probable aplastamiento del concreto en estas nuevas zonas. Se debe notar que la naturaleza cíclica reversible de las fuerzas aplicadas en los ganchos tendría asociado un deterioro de la capacidad resistente del concreto en compresión en la vecindad del gancho. Este deterioro provoca incrementos de deslizamientos en la barra, así como disminución de su capacidad resistente en tensión o compresión.

Los resultados aquí presentados muestran que el anclaje del lecho inferior de la conexión trabe-columna del eje central, tiene un comportamiento diferente al de una conexión monolítica diseñada de acuerdo a requisitos mínimos de reglamentos. Estas diferencias son los deslizamientos de este anclaje ya comentados, así como la característica observada de deficiencias para desarrollar el momento resistente de la sección crítica. Por ejemplo, en el primer nivel, para valores de $D r$ menores que $1.5 \%$, el refuerzo del lecho inferior de la conexión no alcanzó la fluencia, y por tanto su capacidad resistente a flexión. Este comportamiento, el hecho de que durante el ensaye este refuerzo en el segundo nivel no alcanzó la fluencia, así como los deslizamientos mencionados, indican que la conexión trabe-columna del eje central, cuyo refuerzo del lecho inferior no tuvo suficiente longitud de desarrollo, no es capaz de lograr un comportamiento del tipo requerido en un marco dúctil.

La fig. 6 muestra la ubicación del deformímetro instalado en el estribo complementario de la conexión trabe-columna del eje central del nivel 1, en la dirección perpendicular del ensaye, así como las deformaciones medidas en este deformímetro. La fig. 6 muestra que durante todo el ensaye las deformaciones en el estribo instrumentado fueron menores que el de fluencia. Para las conexiones del nivel 2 no se obtuvieron registros porque los deformímetros instalados en esta conexión no funcionaron.

La fig. 7 describe una posible trayectoria de cargas en el nudo trabe-columna para el caso de momento positivo en una conexión monolítica, obtenida mediante la aplicación de un modelo simple de puntal y tirante (Park y Paulay, 1975). Los resultados del análisis de estas cargas se pueden emplear para evaluar el comportamiento de la conexión prefabricada en estudio. La fig. 7a muestra fuerzas actuantes y agrietamientos típicos en una conexión monolítica. La fig. 7b muestra el modelo del puntal y tirante para dicha conexión. Empleando este modelo en la conexión del espécimen, fig. 7c, se observa que el elemento que debe soportar la tensión, Te, son los estribos complementarios. Sin embargo, como se ha demostrado, ocurren deslizamientos importantes en los ganchos del refuerzo inferior de la viga en la zona de la conexión, lo que indica que los estribos complementarios en la conexión no son capaces de desarrollar valores apreciables de la mencionada fuerza Te. El modelo sencillo mostrado en la fig. 7 ayuda a explicar el comportamiento inadecuado de la conexión en estudio. 


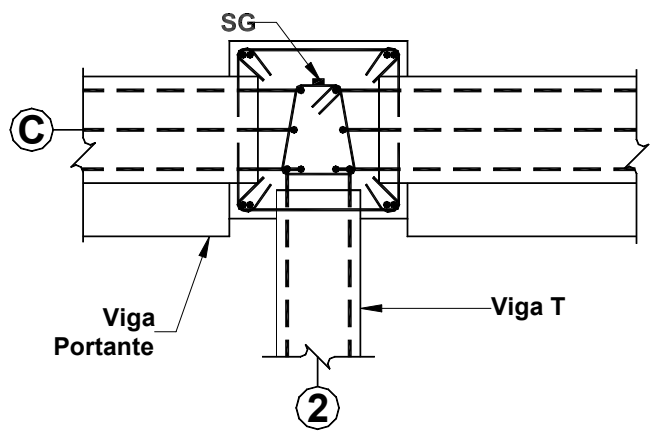

a) Ubicación de deformímetro instrumentado

Nivel 1

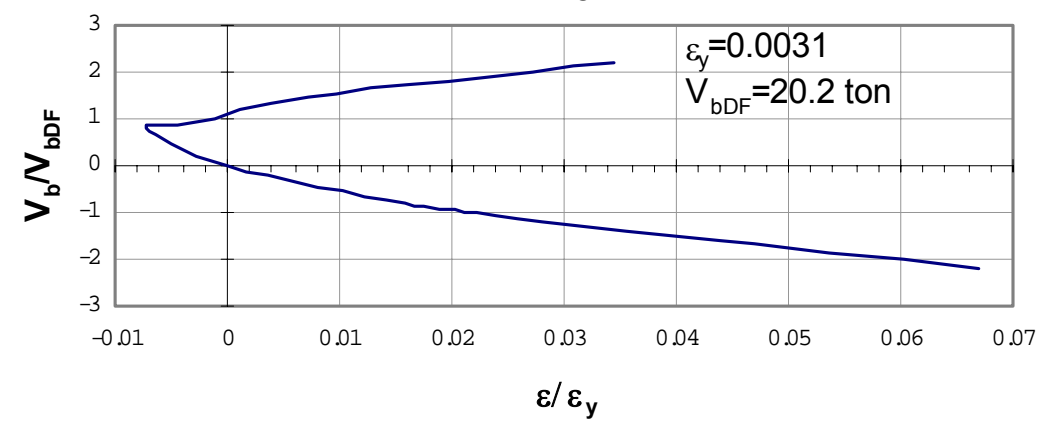

b) Deformaciones medidas

Figura 6. Ubicación y deformaciones medidas en estribo complementario

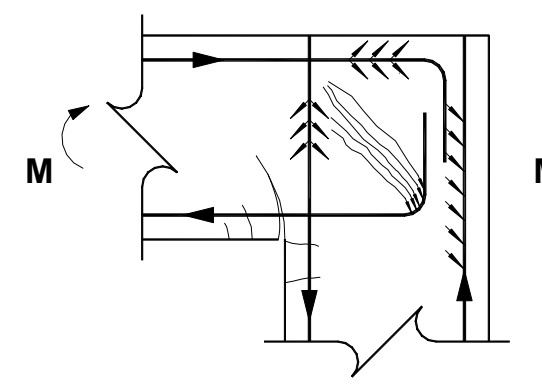

$-1$

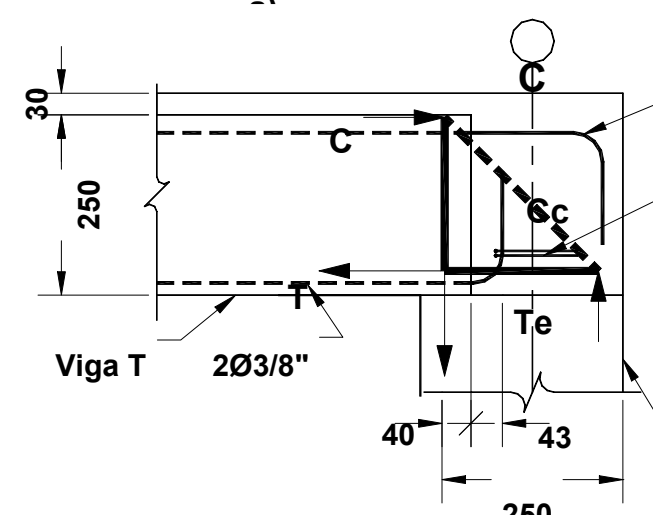

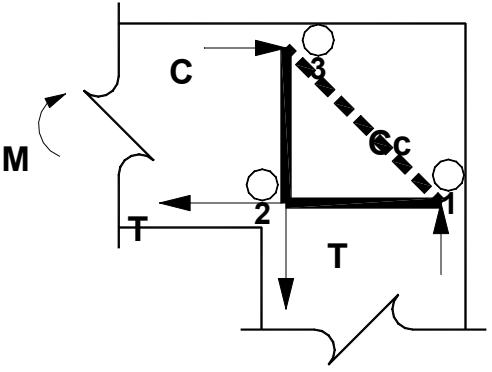

h)

$4 \varnothing 3 / 8 "$ refuerzo

viga $T$

2EØ1/4"

complementarios

n)

Figura 7. Modelo de puntal y tirante para una conexión trabe-columna con momento positivo 
El comportamiento de barras de refuerzo longitudinal en forma de gancho, embebidas en concreto y con longitud insuficiente de desarrollo, ha sido estudiado en la literatura, tanto para los casos de cargas monotónicas (Minor y Jirsa, 1975), como para cargas cíclicas reversibles (Eligehausen et al., 1982). Los resultados de estas investigaciones son congruentes con los de este estudio, en el sentido de que este tipo de refuerzo cuando no cumple requisitos de anclaje de reglamentos, presenta deslizamientos importantes, aún antes de alcanzar la fluencia.

\section{COMPORTAMIENTO DEL SISTEMA DE PISO OBSERVADO EN EL ENSAYE Y EVALUACIÓN DE LA CAPACIDAD RESISTENTE A FUERZAS LATERALES EN SU PLANO}

\section{Características del sistema de piso del espécimen}

Las características generales del sistema de piso del espécimen han sido descritas en el artículo compañero de éste (Rodríguez y Blandón, 2003). En lo que sigue se describen algunas características adicionales del sistema de piso, que son de interés para la evaluación del comportamiento de éste durante el ensaye.

El sistema de piso consistió en vigas tipo T para el eje central del espécimen, así como tipo doble T para la parte restante del sistema de piso (Rodríguez y Blandón, 2003). Además, se construyó un firme de espesor $30 \mathrm{~mm}$, con una rugosidad promedio de alrededor de $3 \mathrm{~mm}$ con el fin de conectar las vigas $\mathrm{T}$ o doble $\mathrm{T}$ con el firme. En este firme se colocó una malla

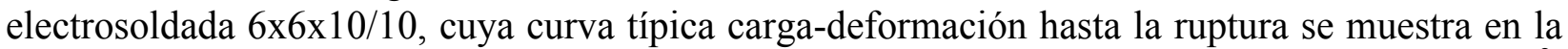
fig. 8. Los esfuerzo de fluencia y de ruptura medidos en esta malla fueron 4010 y $7200 \mathrm{~kg} / \mathrm{cm}^{2}$, respectivamente. El diseño de esta malla para el firme siguió los requisitos de diseño por temperatura y contracción especificados por las NTC96, los cuales son semejantes a los del ACI 318-02. Esta malla en el firme de $30 \mathrm{~mm}$ de espesor corresponde a una cuantía de refuerzo igual a 0.002 . Se debe mencionar que los requisitos de diseño por cortante del firme, de acuerdo con las NTC96, no rigieron el diseño de éste.

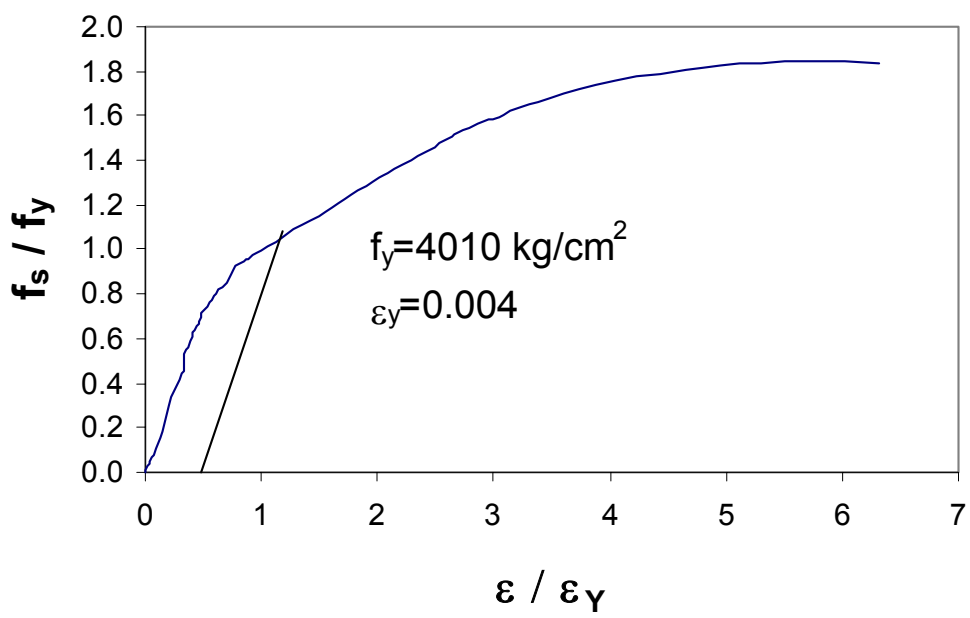

Figura 8. Curva experimental esfuerzo deformación para la malla electrosoldada del espécimen 
B

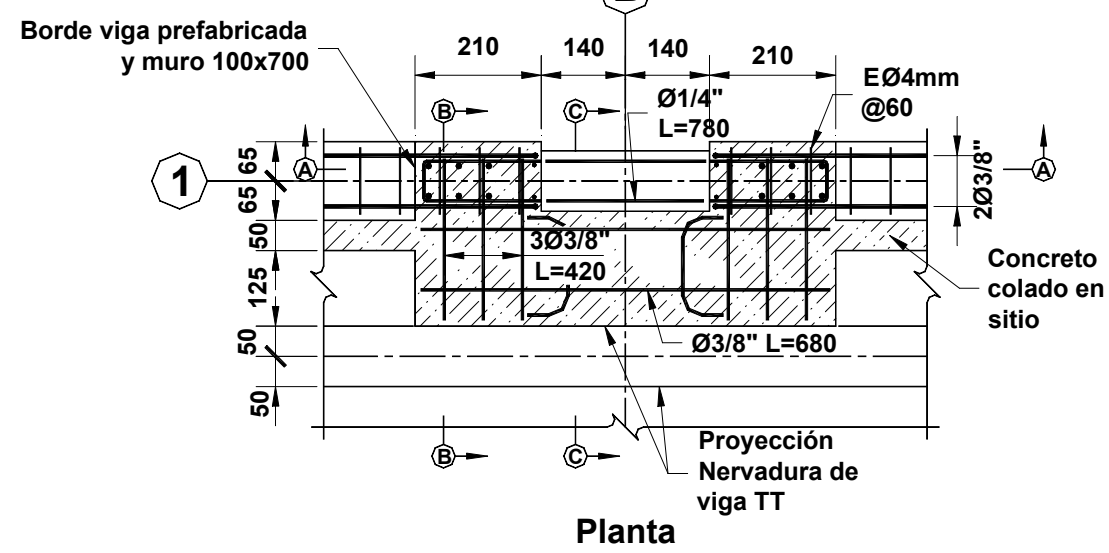

B

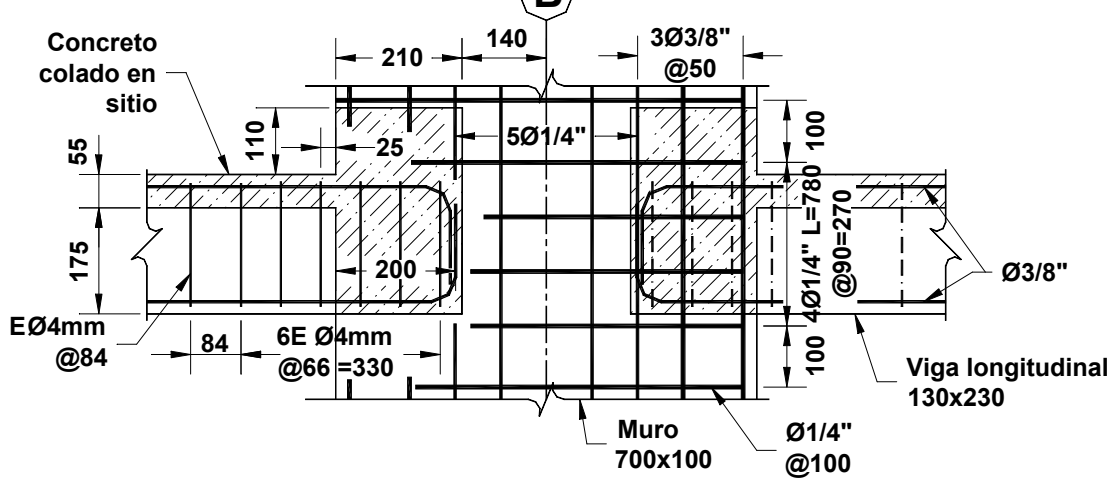

Sección A-A

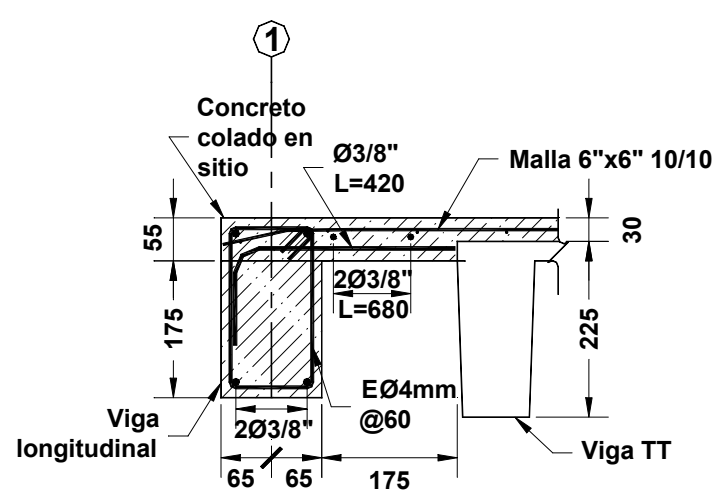

Sección B-B

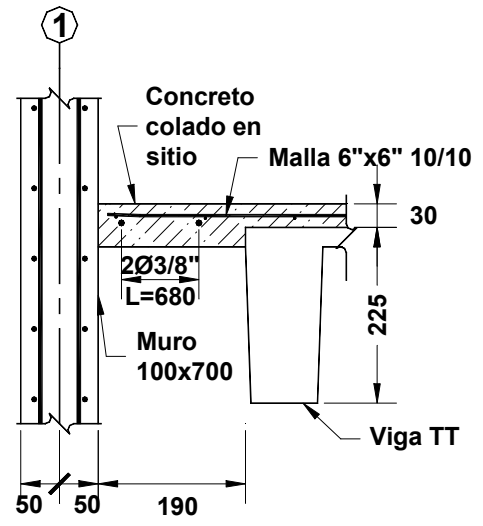

Sección C-C

- Dimensiones en milímetros

- Concreto colado en sitio

Figura 9. Detalles de conexiones entre elementos prefabricados y muros estructurales

La conexión del sistema de piso a los muros del espécimen, eje B en la planta de la fig. 9, se resolvió aumentando el espesor del firme en esta zona, lo cual se logró dejando sin colar la 
aleta de la viga doble $\mathrm{T}$ en un ancho igual al del muro, por lo que fue posible colocar barras de refuerzo $\phi 3 / 8$ " que sobresalieron del muro hacia el sistema de piso (fig. 9). Se debe observar que existe una sección crítica del sistema de piso sin este refuerzo, la que se ubica en la sección del firme a cara de nervadura de la viga doble $\mathrm{T}$, la que se puede observar en las secciones B-B y C$\mathrm{C}$ de la fig. 9. Como se describe posteriormente, en esta sección crítica ocurrió la separación del sistema de piso respecto al muro, con la fractura de la malla del firme.

Se debe mencionar que en nuestro país, en la construcción del tipo de sistema de piso prefabricado que aquí se estudia, existe la práctica de conectar las alas de las vigas $\mathrm{T}$ o doble $\mathrm{T}$ soldando elementos metálicos embebidos en el concreto, con el fin de lograr en estas vigas desplazamientos verticales uniformes al momento de ser cargadas. En el caso del espécimen ensayado estos elementos metálicos no se instalaron en las vigas del sistema de piso del espécimen, con el propósito de estudiar el comportamiento del sistema de piso sin la conexión entre las alas de las vigas $\mathrm{T}$ y doble $\mathrm{T}$. Lo que sí se consideró en la construcción del sistema de piso del espécimen es una solución que no es común en la práctica en México, pero que los autores consideran favorable para la respuesta sísmica del sistema de piso. Esta solución se ilustra en la fig. 10 y consiste en tratar de lograr una mejor continuidad entre las vigas $\mathrm{T}$ o doble $\mathrm{T}$ del sistema de piso y las vigas portantes. Como se aprecia en la fig. 10, se abrió una abertura en la aleta de la viga doble $\mathrm{T}$ con el fin de colar concreto en sitio de manera de lograr una mayor integración del firme con las trabes portantes.

La fig. 11 muestra una vista general del sistema de piso para el segundo nivel del espécimen antes de colar el firme.

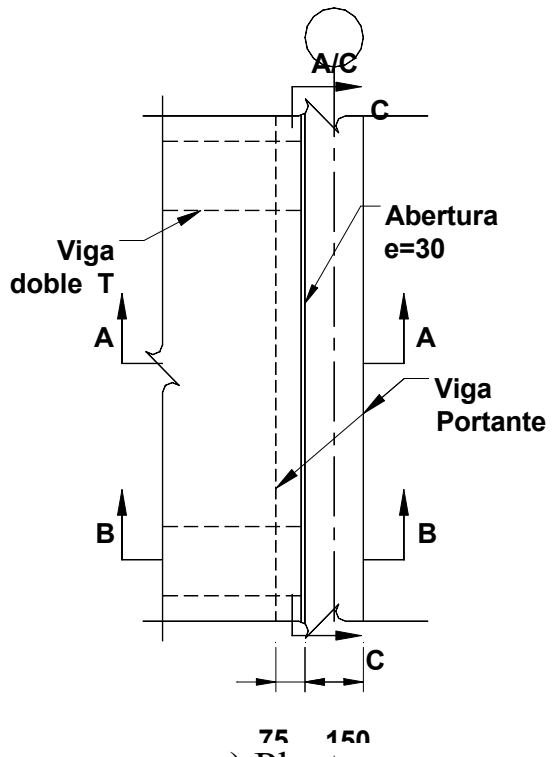

a) Planta

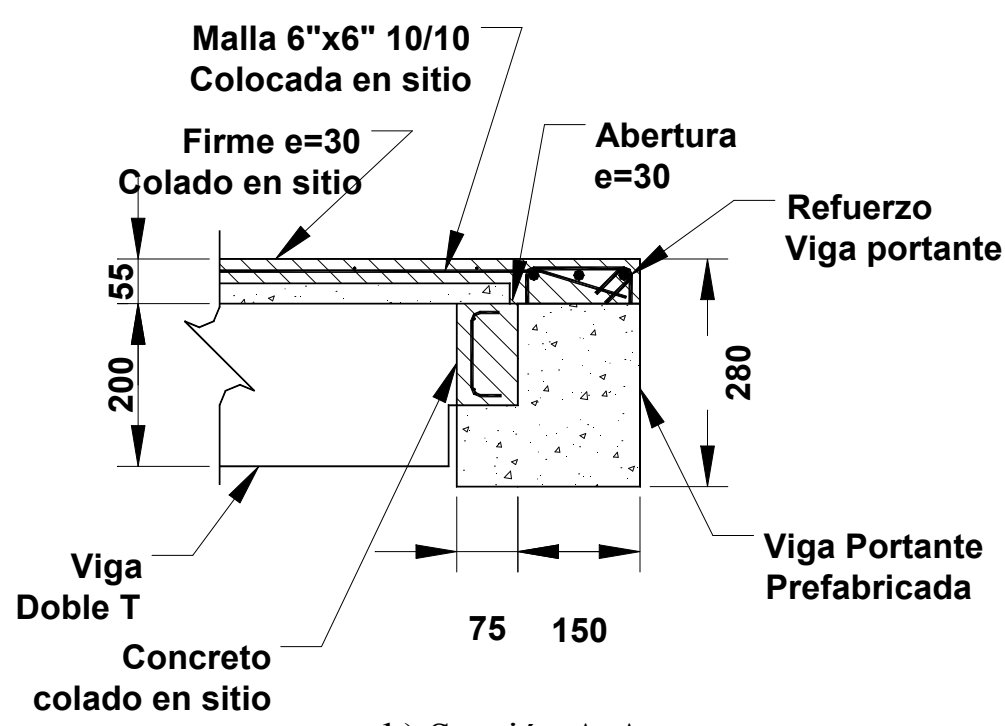

b) Sección A-A

Figura 10. Detalles de continuidad entre sistema de piso prefabricado y trabes portantes, dimensiones en milímetros 


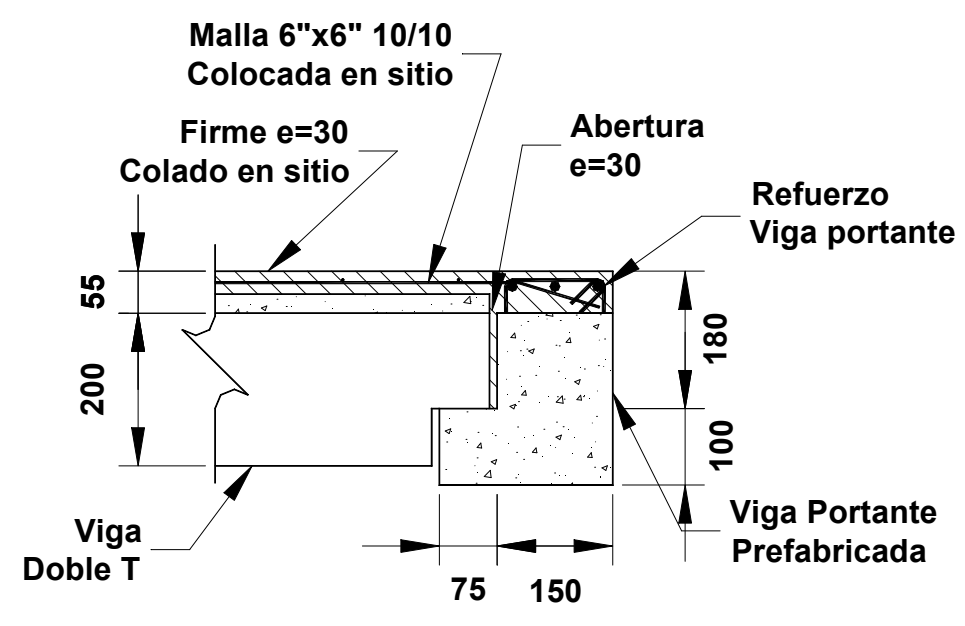

c) Sección B-B

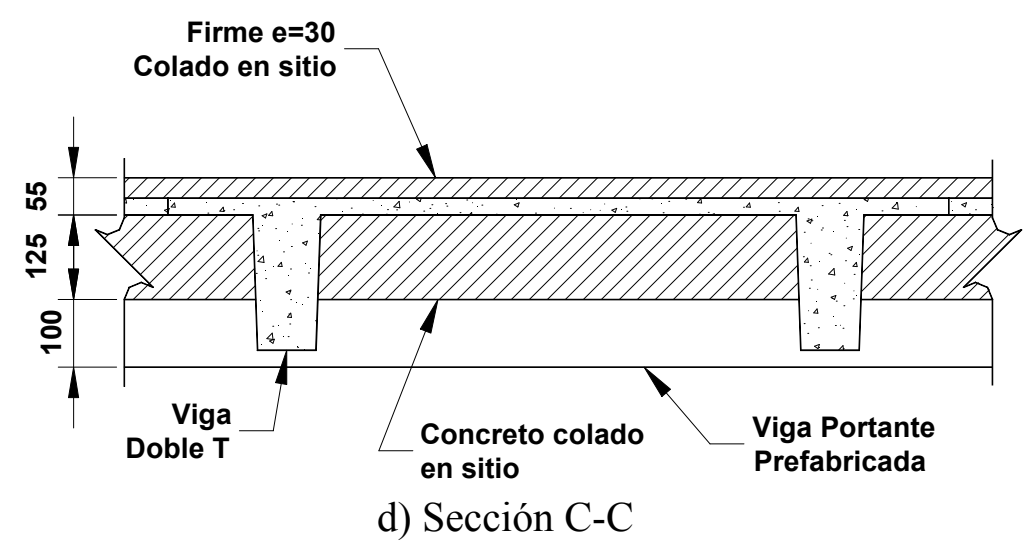

Figura 10. Detalles de continuidad entre sistema de piso prefabricado y trabes portantes, dimensiones en milímetros (continuación)

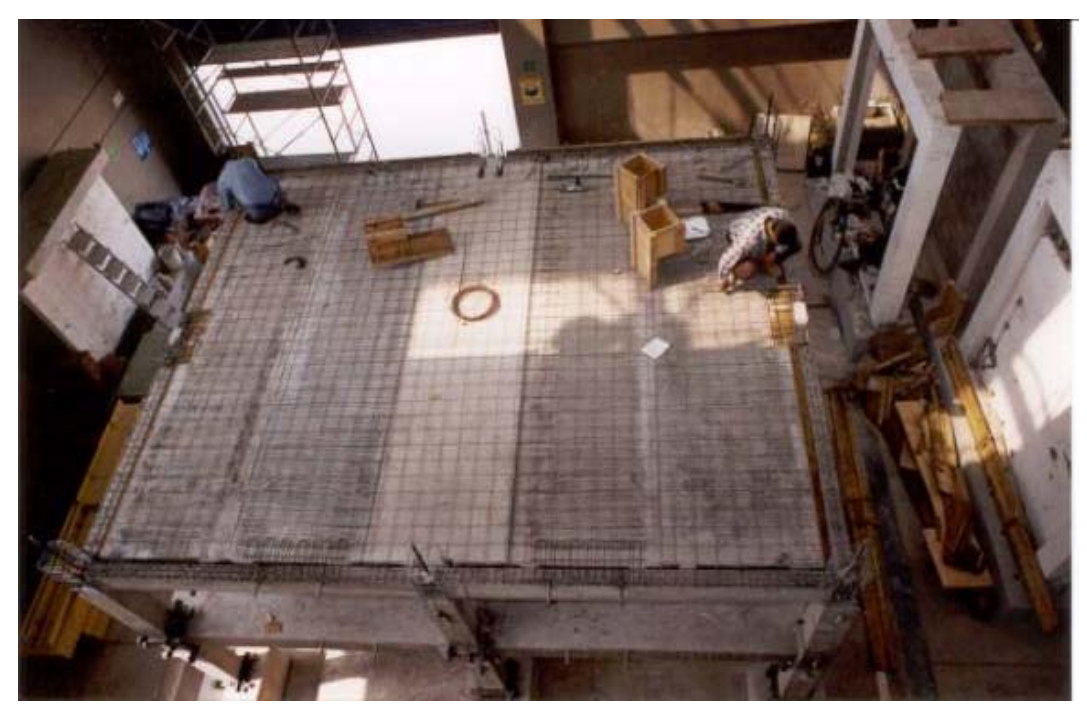

Figura 11. Vista general del nivel 2 antes de colar el firme 


\section{Comportamiento observado en el ensaye}

De acuerdo con lo observado en el ensaye del espécimen, el inicio del agrietamiento en el sistema de piso y probable fractura de la malla del firme se presentó en la sección crítica en la vecindad del muro descrita anteriormente, en el intervalo de cortantes máximos aplicados, $V_{b} / V_{b D F}$, de $1.4 \mathrm{a}$ 2.1, es decir, entre 28.3 y 42.6 ton (fig. 12). En esta sección crítica el ancho de grieta máximo observado en el firme fue igual a alrededor de $10 \mathrm{~mm}$, cuando el espécimen alcanzó un valor del desplazamiento relativo global, $D r$, cercano a 0.02 . Este importante agrietamiento se le puede asociar al inicio de la inestabilidad de los muros fuera del plano, originado por el inicio del pandeo del refuerzo longitudinal de éstos para este nivel de deformación lateral (Rodríguez y Blandón, 2003).

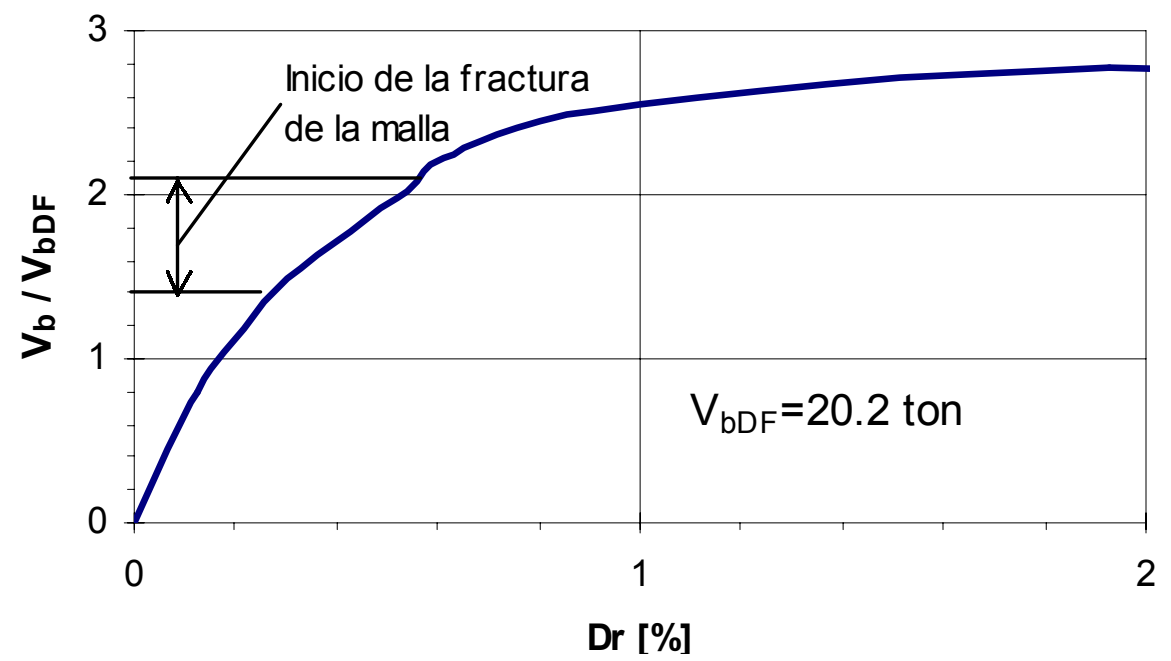

Figura 12. Intervalo de la respuesta del espécimen en el que se observó el inicio de la fractura de la malla

\section{Evaluación de la capacidad resistente del sistema de piso de piso (diafragma) a fuerzas laterales en su plano}

\section{Evaluación de la resistencia del diafragma de acuerdo a criterios de reglamento}

La resistencia por cortante del firme del diafragma del espécimen de acuerdo con el ACI318-02 se basa en el concepto de cortante por fricción, en el cual se ignora la contribución del concreto a esta resistencia. La resistencia nominal por cortante del firme, $V_{n}$, se define como

$V_{n}=\rho_{n} \cdot f y \cdot A_{c v}$

donde $\rho_{n}=$ cuantía de refuerzo transversal en el firme, $f y=$ esfuerzo de fluencia del refuerzo distribuido en el firme y $A_{c v}=$ área neta de la sección transversal. 
Los parámetros $\rho_{n}$ y $f y$ en el espécimen fueron iguales a 0.002 y $4010 \mathrm{~kg} / \mathrm{cm}^{2}$, respectivamente. La definición de $A_{c v}$ se interpreta considerando el ancho total en el espécimen $(500 \mathrm{~cm})$ y el espesor del firme, lo que lleva a $1500 \mathrm{~cm}^{2}$. Empleando estos valores y la ecuación (1) se encuentra $V_{n}=12.0$ ton. De acuerdo con la distribución de fuerzas laterales en el espécimen a este valor de cortante le corresponde un valor de cortante basal igual a 36.0 ton. Como se aprecia en la fig. 12 este valor cae dentro del intervalo de cortante basal en el espécimen en que se observó el inicio del agrietamiento del firme y probable fractura de la malla electrosoldada. Sin embargo, de acuerdo con el ACI 318-02, el refuerzo por cortante se debe colocar en todo el peralte del diafragma (5.0 m en el espécimen), lo que no es congruente con lo observado en el espécimen, el cual mostró evidencias de concentraciones de fuerzas en zonas reducidas del diafragma como se comenta posteriormente.

Es de interés comentar los requisitos de las NTC96 para el diseño por cortante de diafragmas en edificaciones, y en particular su aplicación al espécimen estudiado en esta investigación. Tanto las NTC96 como las Normas Técnicas Complementarias para Estructuras de Concreto de 2002 (NTC02) especifican que los diafragmas se dimensionen con los criterios para vigas. De acuerdo con estos requisitos, la resistencia al cortante de diafragmas en edificaciones está dada por la suma de las contribuciones del concreto y del acero. En el caso del espécimen, la aplicación de estos requisitos para la primera contribución lleva a un cortante resistente en el diafragma que tiene asociado un cortante basal en el espécimen igual a 60 ton. La contribución del acero de refuerzo del espécimen de acuerdo con estos requisitos tendría asociado un cortante basal de igual valor al anteriormente calculado de acuerdo con el ACI 318-02, es decir 36 ton. La suma de ambas contribuciones lleva a un cortante basal de 96 ton, valor bastante mayor que el máximo observado en el ensaye del espécimen. Esto indica que cuando se emplean los requisitos de diseño de las NTC 96 y NTC02, sus resultados están del lado de la inseguridad en lo que se refiere a la predicción de resistencia por cortante de diafragmas en el espécimen, lo que sugiere una situación similar en edificaciones prefabricadas con características comparables a las del espécimen.

\section{Evaluación de la capacidad resistente del sistema de piso de piso (diafragma) a fuerzas laterales en su plano de acuerdo con el modelo del puntal y tirante}

El modelo del puntal y tirante (Schlaich, 1987) es una herramienta útil y a la vez simple, la cual cuando se emplea con criterios razonables puede llevar a una buena estimación de la resistencia requerida, así como a identificar los elementos o secciones críticas en una estructura. Esta herramienta se aplicó para el estudio de la capacidad resistente del diafragma a fuerzas en su plano. Como se describe en detalle en (Rodríguez y Blandón, 2002), se estudiaron varios modelos de puntal y tirante, y se empleó el modelo que llevaba al mínimo trabajo interno. Este criterio de selección de un modelo con el mínimo trabajo interno lleva a mejorar la predicción de la capacidad resistente, ya que el modelo del puntal y tirante corresponde al método del límite inferior. El modelo seleccionado se ilustra en la fig. 13.

La fig. 14 muestra los tirantes y puntales superpuestos con un esquema del agrietamiento en el piso del segundo nivel observado al final del ensaye. La fig. 15 muestra detalles de la zona 
crítica del sistema de piso, la que ha sido comentada anteriormente, así como fuerzas actuantes en esta zona para el modelo ilustrado en la fig. 14. El análisis de fuerzas actuantes en los puntales y tirantes de este modelo indicó que los elementos críticos son los tirantes, en particular los que llegan a los muros y que se muestran en la fig. 14 (Rodríguez y Blandón, 2002).

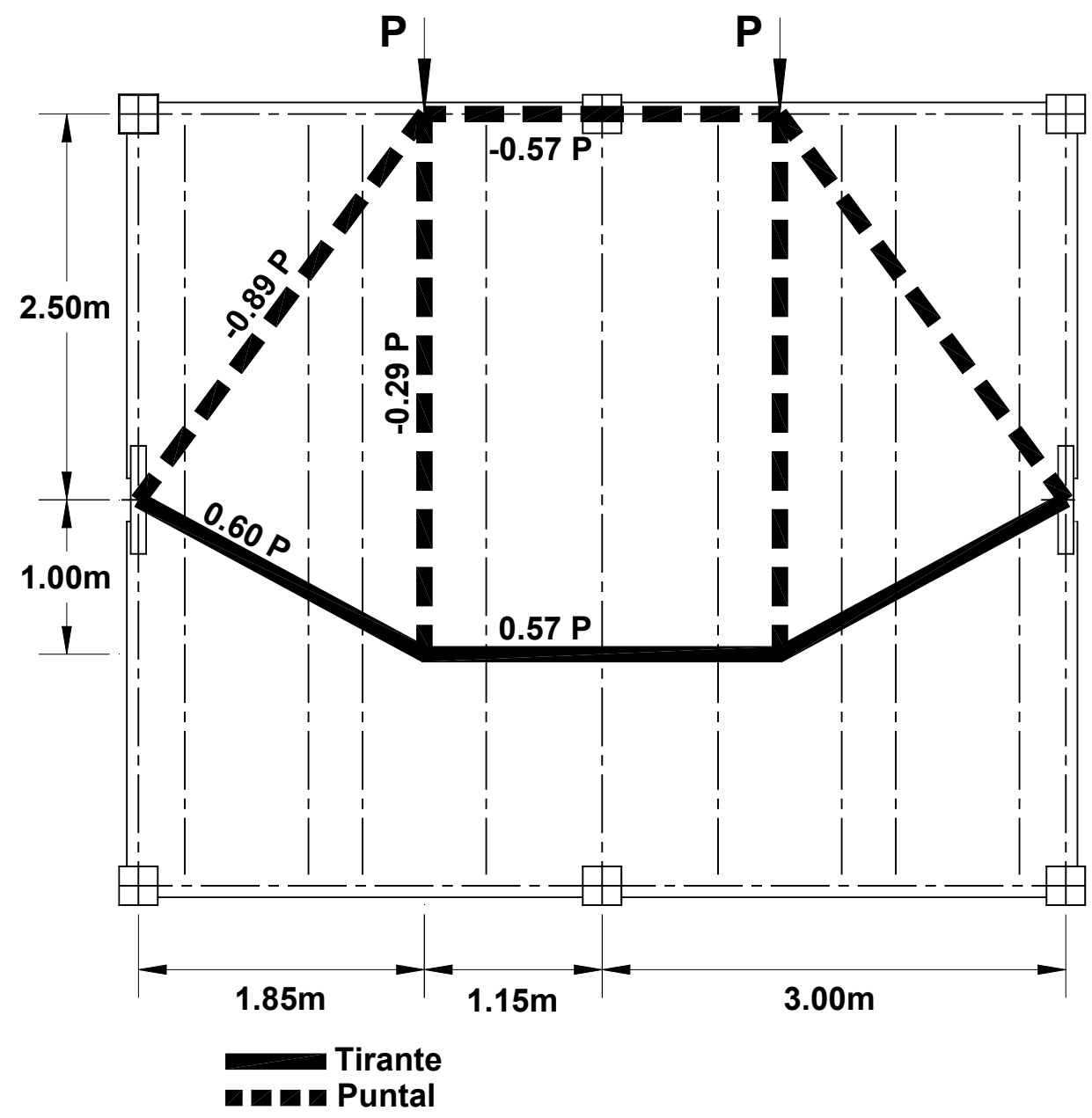

Figura 13. Fuerzas actuantes en el modelo de puntal y tirante para el sistema de piso del espécimen

El agrietamiento mostrado en la fig. 14 indica que la losa se fracturó en la zona cercana del muro. Esta fractura se ubicó en una grieta en el firme en la vecindad con los muros, en la sección transversal del firme donde el concreto colado en sitio cambia de espesor de $55 \mathrm{~mm}$ a 30 $\mathrm{mm}$, ver secciones B-B y C-C de la fig. 9. Esta sección crítica se identifica como $p q$ en la fig. 15. En la evaluación de la resistencia a tensión del tirante en esta sección crítica se consideró que la tensión es resistida sólo por la malla electrosoldada, y que los puntales son resistidos por el firme con los anchos respectivos. La evaluación de la capacidad resistente del modelo de puntal y tirante que se muestra en la fig. 14 indicó que esta capacidad resistente está asociada a cortantes basales en el espécimen iguales a 20.5 ton y 36.8 ton, cuando se considera la fluencia y la fractura de la malla, respectivamente. Este intervalo de predicción de resistencia es una estimación algo 
conservadora de la resistencia del diafragma a fuerzas en su plano observada durante el ensaye, ver fig. 12. Parte de esta estimación conservadora de la predicción de la resistencia del diafragma en su plano se puede explicar considerando la naturaleza del modelo del puntal y tirante, el cual por corresponder a un criterio de límite inferior de la teoría del análisis plástico debe llevar a una estimación conservadora de la resistencia.

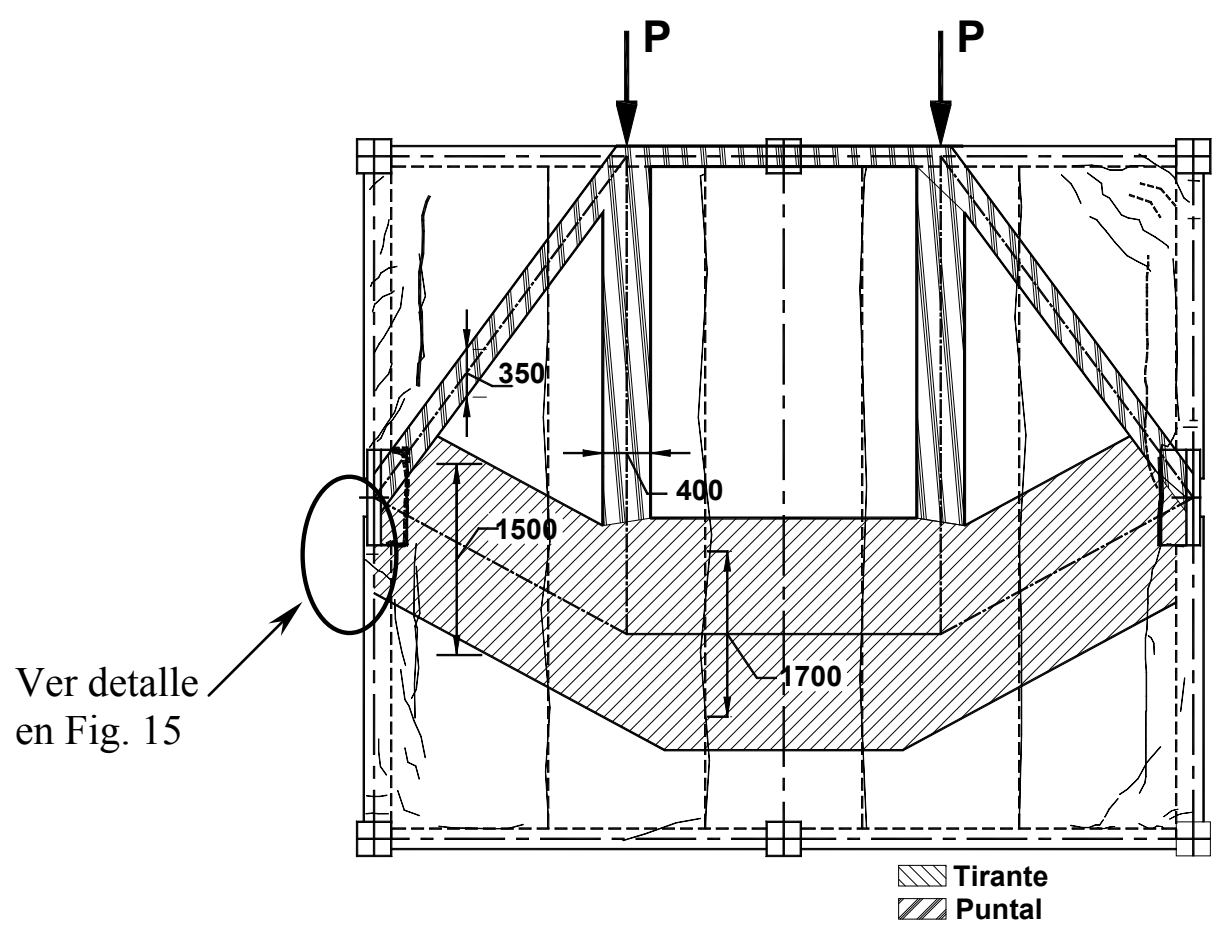

Figura 14. Modelo de puntal y tirante en el sistema de piso y agrietamiento observado al final del ensaye (dimensiones en milímetros)

Es de interés que después del inicio de la fractura en la sección crítica del diafragma en la vecindad del muro, durante el ensaye no se observó que la capacidad del espécimen decreciera súbitamente, además, el agrietamiento mostrado en la fig. 14 indica que éste no se presentó sólo en la vecindad del muro. Dado que después de la fractura de la malla en la sección crítica pq mostrada en la fig. 15, esta sección no podría resistir alguna fuerza en tensión, el comentario anterior sugiere que después de la mencionada fractura, sería necesaria la existencia de un nuevo mecanismo que pueda seguir resistiendo las fuerzas actuantes en el plano del diafragma. En la fig. 16 se muestra el nuevo mecanismo que se postula, el cual se considera que aparece después de la ruptura de la malla en la sección crítica mencionada. La trayectoria de cargas que se postula requiere que el agrietamiento en el diafragma se produzca en una zona alejada de los muros, lo que es congruente con lo observado en el ensaye (fig. 14). Como se aprecia en la fig. 16, en este nuevo modelo las trabes laterales reciben la carga del firme y éstas a su vez la transmiten a los muros. De acuerdo con un análisis de fuerzas actuantes y resistentes en los puntales y tirantes del nuevo modelo, el cortante basal asociado a la condición crítica en este modelo es igual a 28.0 ton. Aun cuando este valor es una estimación conservadora respecto al máximo observado en el espécimen, el nuevo modelo propuesto ayuda a explicar el motivo por el cual la capacidad lateral 
del espécimen no se afectó drásticamente después de la fractura de la malla del firme en la vecindad del muro.

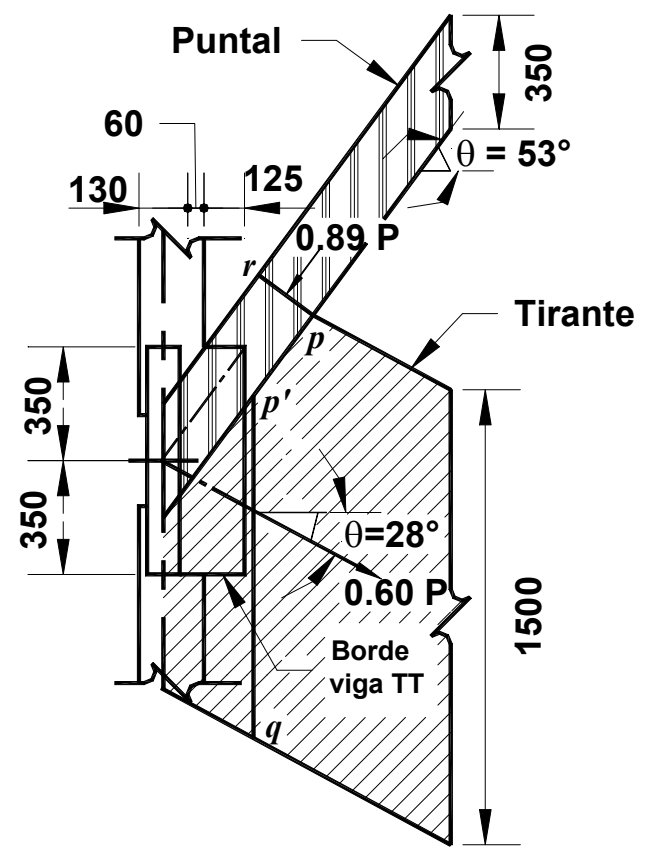

Figura 15. Detalle de zona crítica (dimensiones en milímetros)

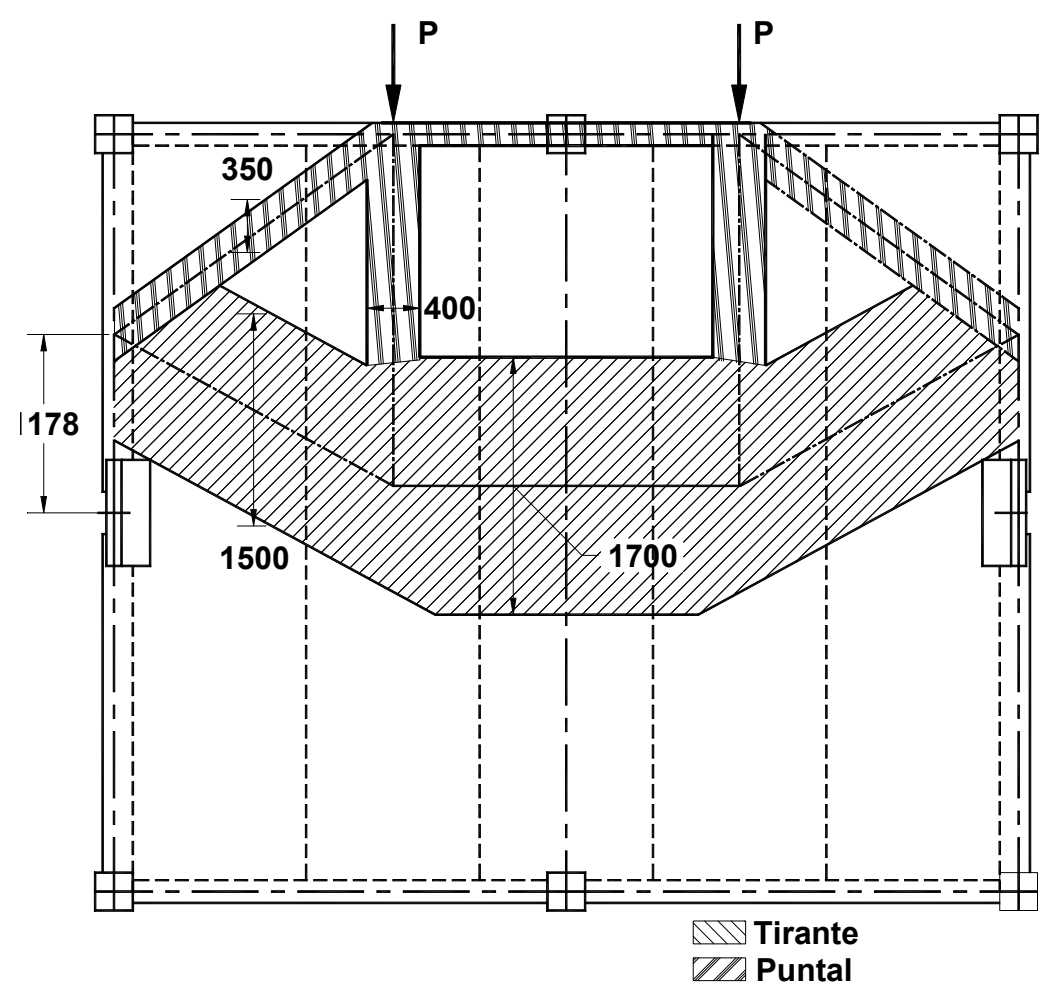

Figura 16. Modelo de puntal y tirante en el sistema de piso después de la fractura de la malla en la zona crítica muro-sistema de piso 
El modelo empleado en este estudio, para la evaluación de la capacidad resistente del diafragma a fuerzas en su plano, es muy simple para incluir todas las contribuciones y características de la capacidad resistente del sistema de piso, pero logra explicar de manera aceptable el comportamiento observado durante el ensaye, así como pone en evidencia los aspectos críticos de este comportamiento. Como se ha mostrado para el espécimen, el modelo empleado del puntal y tirante permite que el diseñador pueda evaluar los requerimientos de acero de refuerzo mínimo en las secciones críticas del firme del diafragma. Este acero de refuerzo en estas secciones críticas no podría ser identificado con procedimientos como los del ACI 318-02, menos con los de las NTC96 y NTC02, como ya se ha mostrado. Esto se debe a que estos procedimientos ignoran las posibles concentraciones de deformaciones en las conexiones entre el diafragma y elementos verticales de un sistema estructural resistente a sismos (tales como columnas y muros).

Evaluación del comportamiento monolítico entre el firme y las trabes prefabricadas del sistema de piso

La fig. 17 ilustra el tipo de instrumentación empleado para medir el desplazamiento relativo entre el firme y los elementos prefabricados del sistema de piso. La forma para medir estos desplazamientos consistió en dejar descubierta la parte superior de los prefabricados en una superficie de $40 \mathrm{~mm}$ x $40 \mathrm{~mm}$, para fijar allí elementos de aluminio que sirvieran de referencia de medición de los micrómetros ubicados en el firme, fig. 17. Este detalle se llevó a cabo en cuatro puntos del sistema de piso por cada nivel del espécimen.

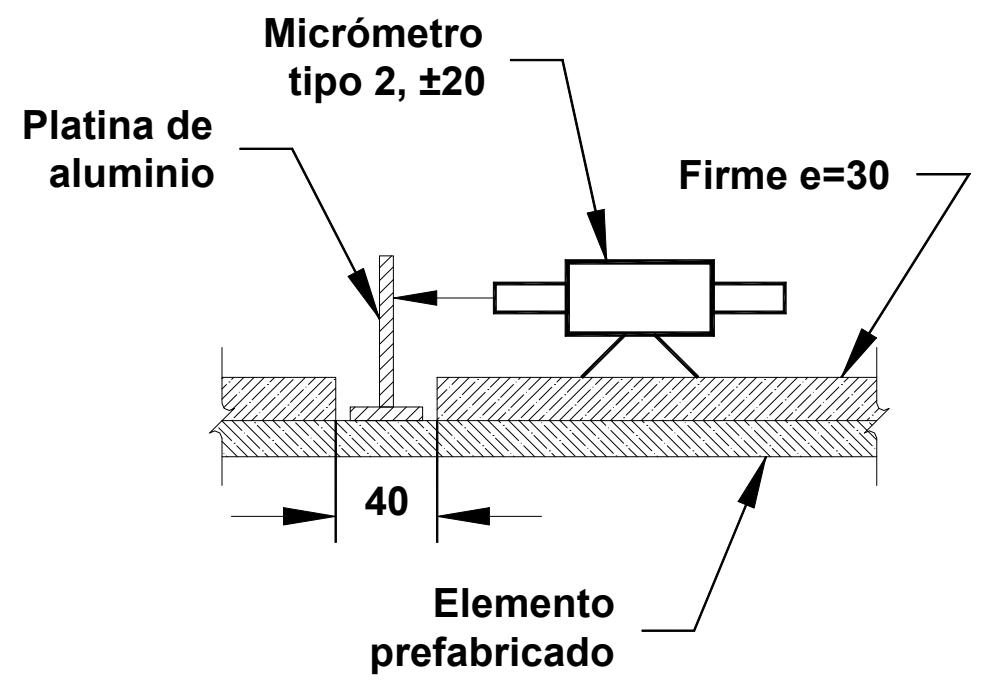

Figura 17. Instrumentación para el estudio del comportamiento del firme (dimensiones en milímetros)

Es relevante comentar las mediciones obtenidas con esta instrumentación durante el ensaye del espécimen ante cargas laterales, ya que requisitos de diseño de las NTC96 y ACI 31802 para lograr un comportamiento monolítico entre el firme y el sistema de piso prefabricado son en general vagos. Por ejemplo, las NTC96 especifican que se debe colocar conectores que 
impidan que el firme se separe de los elementos prefabricados; sin embargo, estas Normas no dan criterios específicos para lograr este objetivo. EL ACI 318-02 permite un trabajo de sección compuesta entre el firme y el piso prefabricado si se cumple un criterio semejante al mencionado de las NTC96, es decir sin un criterio específico de diseño; además, requiere en este caso que la superficie endurecida del piso prefabricado deba tener una "rugosidad intencional"; sin embargo, el ACI 318-02 no da criterios para cuantificar este requisito. Por otro lado, para considerar un comportamiento monolítico entre el firme y el piso prefabricado, las NTC02 especifican una rugosidad mínima de $5 \mathrm{~mm}$, sin hacer referencia a las posibles evidencias observadas que sirvan de bases para estos requisitos.

Las mediciones obtenidas con la instrumentación para medir deslizamientos entre el firme $\mathrm{y}$ el piso prefabricado indicaron que estos deslizamientos no fueron relevantes. Este comportamiento es congruente con el comportamiento observado en el sistema de piso durante el ensaye, en el cual, como se ha descrito, la falla del diafragma estuvo asociada principalmente a la fractura de la malla del firme en las secciones críticas de éste. Debido a este modo de falla, la condición de comportamiento monolítico entre el firme y el piso prefabricado no fue relevante. Como se aprecia en la fig. 8, correspondiente a la curva experimental esfuerzo-deformación de esta malla, la ruptura de ésta está asociada a un modo de falla frágil, ya que las deformaciones de ruptura son bastante menores que las típicas de ruptura en aceros de refuerzo convencionales que se emplean en elementos estructurales.

\section{DISEÑO Y COMPORTAMIENTO DE LA CONEXIÓN ENTRE COLUMNA PREFABRICADA Y CIMENTACIÓN (CANDELERO)}

En el diseño y construcción de cimentaciones en estructuras prefabricadas en nuestro país, una práctica común en la industria de la construcción es el empleo de los llamados candeleros. Estos son elementos prefabricados en forma de zapata con un espacio interior donde se introduce la columna prefabricada como se aprecia en la fig. 18, para posteriormente colar con mortero expansivo la junta en la columna embebida.

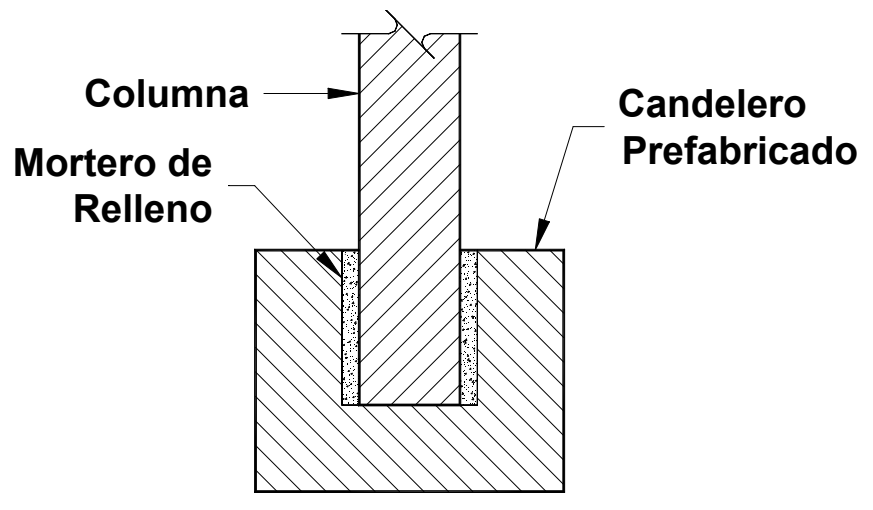

Figura 18. Cimentación tipo "candelero" 
Aún cuando esta solución estructural se emplea en construcciones en zonas sísmicas en nuestro país, las NTC96, así como la propuesta de Normas NTC02, no tienen requisitos de diseño para este tipo de cimentación. Es de interés que incluso en países con zonas de alta sismicidad, tales como Japón y USA tampoco cuentan con normatividad específica para este tipo de cimentación.

La fig. 19 (Osanai et al., 1996) muestra un diagrama de cuerpo libre en el candelero y columna sometida a cargas laterales. En esta figura las fuerzas $C$ y $C_{1}$ son las reacciones horizontales en compresión en el candelero, $\mathrm{R}$ es la reacción vertical en el fondo de la columna, $\mathrm{y}$ las fuerzas $F_{1}, F_{2}$ y $F_{3}$ son fuerzas de fricción. Además, como se observa en la fig. 19 para lograr el equilibrio de la fuerza $C$ en el candelero, ésta se debe ser resistir con fuerzas de tensión en el acero de refuerzo del candelero.

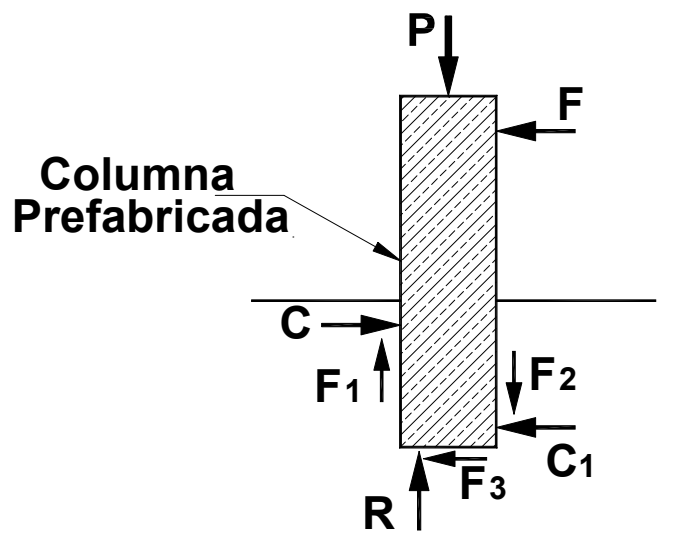

a) Columna

Acero de refuerzo en la cimentación

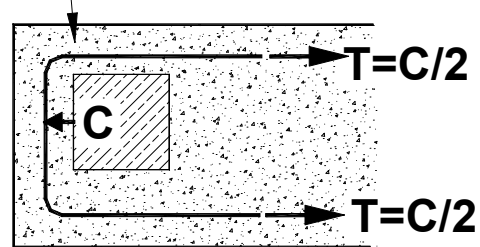

b) Candelero

Figura 19. Fuerzas actuantes en columna prefabricada y candelero

Osanai et al. (1996) han efectuado en laboratorio ensayes de conexiones entre columna prefabricada y candelero, las cuales se sometieron a cargas laterales como se indica en la fig. 19. Con base en la información experimental obtenida, estos autores han propuesto un criterio para evaluar la fuerza $C$, con la cual como se indica en la fig. 19, es posible diseñar el acero de refuerzo en el candelero. Además, estos autores sugieren que si la longitud de columna embebida en el candelero es igual o mayor que $1.5 h_{c}$, donde $h_{c}$ es el peralte de la columna, la conexión 
prefabricada se puede considerar como una conexión rígida, aún cuando la parte de columna embebida no cuente con conectores de cortante. En este caso, para el cálculo de la fuerza $C$ estos autores recomiendan el empleo de un coeficiente de fricción igual a 1.0.

En esta investigación, el diseño de los candeleros del espécimen se efectuó siguiendo las recomendaciones de Osanai et al. (1996), y ha sido descrito en detalle en Rodríguez y Blandón (2002). Con base en estas recomendaciones, en el espécimen la longitud de columna embebida en el candelero fue aproximadamente igual a $1.5 h_{c}$. Además, el diseño de la cimentación se efectuó considerando un cortante basal en el espécimen igual a 1.2 veces el cortante basal máximo observado en el ensaye. La inspección de los candeleros al finalizar el ensaye del espécimen mostró evidencias claras de que la conexión columna prefabricada-candelero tuvo un comportamiento de conexión rígida. Además, en esta inspección no se observó agrietamientos en los candeleros que indiquen daños en éstos. Esto indica que el procedimiento de diseño seguido para los candeleros del espécimen se puede considerar adecuado.

\section{CONCLUSIONES}

1. Se ensayó ante cargas laterales cíclicas reversibles una estructura prefabricada de dos niveles construida con el sistema dual. El refuerzo en forma de gancho en el lecho inferior de algunas trabes, en la zona del nudo trabe-columna, no cumplía los requisitos de reglamento para su longitud de desarrollo. Este refuerzo en el nudo contaba con estribos cerrados, práctica que se emplea en México con el objeto de mejorar el problema de falta de adherencia en casos como el mencionado. Durante el ensaye, se midieron deslizamientos importantes de este refuerzo en el nudo, además de que llegó a la fluencia sólo para niveles de deformación lateral del espécimen y de daños considerables. Este comportamiento es congruente con el observado por otros autores en ensayes de barras de refuerzo embebidas en concreto con longitud insuficiente de desarrollo, cuando estas barras fueron sometidas a fuerzas de tensión o compresión del tipo monotónicas o en los casos de cargas cíclicas reversibles. En estos ensayes de otros autores fue notorio que el problema de deslizamiento en las barras estuvo asociado a aplastamiento del concreto en zonas alrededor de la barra, lo que es un comportamiento indeseable en estructuras sismorresistentes. Así mismo, en el ensaye del espécimen se observó que los estribos cerrados mencionados no contribuyeron a mejorar el problema de falta de adherencia del refuerzo al que estaban conectados. Este comportamiento, así como el deslizamiento observado en el refuerzo con problemas de adherencia, indican que este tipo de conexión no se debe usar en estructuras cuyo sistema estructural sismorresistente sea a base sólo de marcos, ya que esta conexión tiene un comportamiento inelástico bastante limitado. Como se ha demostrado en esta investigación, es posible emplear este tipo de conexiones en estructuras prefabricadas sólo cuando el sistema estructural es del tipo dual, es decir cuando se combina muros y marcos, donde la mayor parte de las fuerzas sísmicas son resistidas por los muros estructurales.

2. Las NTC96 y NTC02 especifican que los diafragmas en edificaciones prefabricadas se dimensionen con los criterios para vigas, combinando las contribuciones del concreto y 
del acero de refuerzo. La aplicación de estos requisitos para la evaluación de la resistencia a cortante de los diafragmas del espécimen llevó a valores bastante mayores que los observados en el ensaye. Es decir estos requisitos llevan a resultados del lado de la inseguridad para la predicción de resistencia de diafragmas a fuerzas en su plano. Esto se debe a que, como se demuestra en esta investigación, el mecanismo de resistencia de diafragmas a fuerzas en su plano es diferente al de vigas que siguen las Normas mencionadas.

3. Los requisitos del ACI318-02 para la evaluación de la resistencia a cortante de diafragamas se basan en el concepto de cortante por fricción, en el cual se ignora la contribución del concreto a esta resistencia. De acuerdo con estos requisitos, el cálculo de esta resistencia para los diafragmas del espécimen llevó a resultados que cae dentro de los niveles observados de resistencia de estos diafragmas, asociados al primer agrietamiento y probable fractura de la malla del firme. Sin embargo, con el procedimiento de diseño del ACI318-02 no es posible identificar las secciones críticas de pisos prefabricados, donde son necesarios refuerzos mínimos para resistir las fuerzas en el plano del diafragma. Como ocurrió en el espécimen, estas secciones críticas se ubicaron en el firme en la vecindad con los muros estructurales, mientras que el procedimiento especificado por el ACI318-02 se basa en el concepto de que el acero de refuerzo en toda la sección transversal del diafragma contribuye a su resistencia por cortante.

4. A pesar de algunas limitaciones del modelo del puntal y tirante, éste fue empleado en el espécimen para evaluar la resistencia de los diafragmas a fuerzas en su plano. Los resultados encontrados con este modelo mostraron que su empleo lleva a una estimación razonable y del lado de la seguridad de la cantidad de refuerzo requerida en el firme sobre el piso prefabricado. Este modelo es particularmente útil para identificar las secciones críticas del diafragma donde se concentran las fuerzas actuantes en su plano. Con este modelo y el criterio de diseño por capacidad es posible diseñar estas secciones para que resistan de manera adecuada las fuerzas actuantes en el plano del diafragma.

5. De acuerdo con los resultados de este estudio se recomienda emplear el modelo del puntal y tirante para el diseño de diafragmas que deban resistir fuerzas en su plano, como es el caso de diafragmas en edificios sometidos a acciones sísmicas. Este modelo da resultados bastante mejores que los que se encuentran con el modelo de viga de las NTC96 ó NTC02, e incluso mejores que los resultados que se obtendrían con la aplicación del concepto de cortante por fricción que emplea el ACI318-02.

6. El uso de candeleros como cimentación en la industria de la construcción de estructuras prefabricadas es bastante frecuente en nuestro país. Sin embargo las NTC96 y NTC02 no especifican requisitos de diseño para este tipo de solución estructural. Los candeleros del espécimen fueron diseñados de acuerdo con recomendaciones propuestas por otros investigadores, quienes de acuerdo con resultados encontrados en ensayes de este tipo de cimentación ante cargas laterales cíclicas reversibles propusieron que la 
longitud de columna embebida en el candelero debe ser igual o mayor que $1.5 h_{c}$, donde $h_{c}$ es el peralte de la columna. De acuerdo con estos investigadores con esta dimensión es posible lograr en el candelero un comportamiento de conexión rígida. La inspección de los candeleros al finalizar el ensaye del espécimen mostró evidencias claras de que la conexión columna prefabricada-candelero tuvo un comportamiento de conexión rígida, lo que confirma la recomendación mencionada. Además, en esta inspección no se observó agrietamientos en los candeleros que sugieran daños en éstos. Esto indica que el procedimiento de diseño seguido para los candeleros del espécimen se puede considerar adecuado.

\section{REFERENCIAS}

Alcocer, S, R Carranza y D Perez (2000), "Behaviour of a precast concrete beam-column connection", Memorias, 12th World Conference of Earthquake Engineering, Auckland, New Zealand, CD-ROM.

ACI Committee 318 (2002), "Building code requirements for reinforced concrete (ACI 318-02)", American Concrete Institute, Farmington Hills, Michigan, USA.

Carranza, R (1997), Comunicación personal.

Eligehausen, R, V V Bertero y E Popov (1982), "Hysteretic behavior of reinforcing deformed hooked bars in R/C joints", Memorias, 7th European Conference on Earthquake Engineering, Atenas, septiembre, Vol. 4, pp. 171-178.

Minor, J y J Jirsa (1975), "Behavior of bent bar anchorages”, ACI Journal, abril, pp.141-149.

NTC96 (1996), "Normas técnicas complementarias para diseño y construcción de estructuras de concreto del Reglamento de Construcciones del Distrito Federal”, México D.F.

NTC02 (2002), "Propuesta de normas técnicas complementarias para diseño y construcción de estructuras de concreto del Reglamento de Construcciones del Distrito Federal", México D.F.

Osanai, Y, F Watanabe y S Okamoto (1986), "Stress transfer mechanism of socket base connections whit precast concrete columns", ACI Structural Journal, mayo-junio.

Park, R y T Paulay (1975), Estructuras de concreto reforzado, octava impresión, editorial Limusa, México.

Pérez, D, R Carranza, R Martínez y S Alcocer (1998), “Comportamiento sísmico de un tipo de conexión viga columna de elementos prefabricados de concreto", Memorias, XI Congreso Nacional de Ingeniería Estructural, Monterrey, NL, México, noviembre.

Restrepo, J, R Park y A Buchanan (1989), "Seismic load tests on connections between precast concrete elements", New Zealand Concrete Society, Silver Jubilee Conference. Wairakei, Taupo, Nueva Zelanda.

Rodríguez, M y J Blandón (2002), "Ensayes ante cargas laterales cíclicas reversibles de una estructura prefabricada de concreto reforzado de dos niveles", Series del Instituto de Ingeniería, UNAM, No. 627, marzo. 
Rodríguez, M y J Blandón (2003), "Ensayes ante cargas laterales ciclicas reversibles de un edificio prefabricado de concreto reforzado de dos niveles. Parte I: evaluación del comportamiento global". Revista Ingeniería Sísmica, No. 68, pp 55-92.

Schlaich, J, K Schaefer y M Jennewein (1987), "Towards a consistent design of structural concrete", Journal PCI, Vol. 32, No. 3, mayo-junio, pp. 74-150. 